\title{
River Hydraulics
}

GEOLOGICAL SURVEY WATER-SUPPLY PAPER 1369

This water-supply paper was published as separate chapters $A-E$

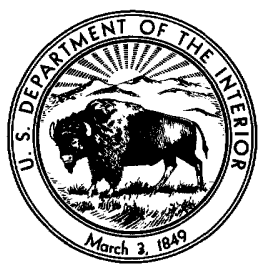


UNITED STATES DEPARTMENT OF THE INTERIOR STEWART L. UDALL, Secretary

\section{GEOLOGICAL SURVEY}

Thomas B. Nolan, Director 


\section{CONTENTS}

[The letters in parentheses designate separately published chapters]

(A) Selected topics of fluid mechanics, by Carl E. Kindsvater

(B) Energy losses associated with abrupt enlargements in pipes, with special reference to the influence of boundary roughness, by Carl $\mathbf{E}$. Kindsvater

(C) Surges in natural stream channels, by S. E. Rantz

(D) Flow through openings in width constrictions, by Jacob Davidian, P. H. Carrigan, Jr., and John Shen

(E) Stream gaging control structure for the Rio Grande conveyance channel near Bernardo, New Mexico, by D. D. Harris and E. V. Richardson 


\section{Selected Topics}

Of Fluid Mechanics

GEOLOGICAL SURVEY WATER-SUPPLY PAPER 1369-A

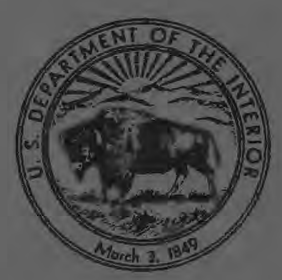




\section{Selected Topics \\ Of Fluid Mechanics}

By CARL E. KINDSVATER

$\begin{array}{llllllllllllllllll}R & I & V & E & R & H & Y & D & R & A & \text { U } & \text { L } & \text { I } & \text { C } & S\end{array}$

GEOLOGICAL SURVEY WATER-SUPPLY PAPER 1369-A

$A$ discussion of the concepts of fluid mechanics which are the foundations of river hydraulics

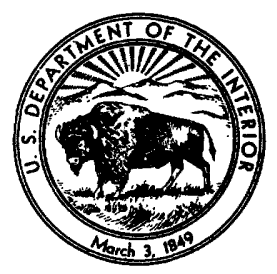




\title{
UNITED STATES DEPARTMENT OF THE INTERIOR FRED A. SEATON, Secretary
}

\author{
GEOLOGIGAL SURVEY \\ Thomas B. Nolan, Director
}




\section{CONTENTS}

\begin{tabular}{|c|c|}
\hline & Page \\
\hline Abstract.-.-. & 1 \\
\hline atroduction.... & $\boldsymbol{2}$ \\
\hline and basic concepts. & $\boldsymbol{z}$ \\
\hline units & 2 \\
\hline dary conditions & $\mathbf{2}$ \\
\hline Fluid properties. & 3 \\
\hline Motion (flow) properties & 3 \\
\hline Force properties. & 4 \\
\hline Ratios and coefficients & 4 \\
\hline Mathematical symbols & 5 \\
\hline Basic concepts of mechanics & 5 \\
\hline Basic concepts of fluid mechanics. & 7 \\
\hline 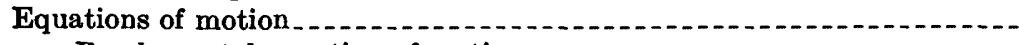 & 10 \\
\hline Fundamental equation of motion & 10 \\
\hline luid motion & 10 \\
\hline Kinematics of fluid motion & 11 \\
\hline Equations for a simple fluid motion & 12 \\
\hline Hydrostatics. & 16 \\
\hline 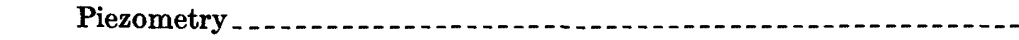 & 18 \\
\hline One-dimensional equation & 20 \\
\hline Equation of continuity & 22 \\
\hline Discharge equation & 23 \\
\hline 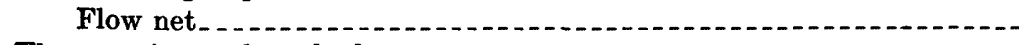 & 24 \\
\hline method.-1. & 28 \\
\hline shear & 29 \\
\hline and the theory of similitude & 29 \\
\hline Influence of a free surface & 32 \\
\hline Recapitulation & 33 \\
\hline Example & 34 \\
\hline (n-1 & 37 \\
\hline e Bernoulli equation & 37 \\
\hline uation. & 40 \\
\hline ation & 42 \\
\hline iform and gradually varied flow & 46 \\
\hline Characteristics of uniform flow & 46 \\
\hline The Chezy equation & 47 \\
\hline Alternate derivation of the Chezy equation & 48 \\
\hline Gradually varied flow in open channels. & 50 \\
\hline
\end{tabular}

\section{ILLUSTRATIONS}

[Plates follow p. 52]

Plate 1. Flow through a two-dimensional orifice, piezometers in pipes and open channels, and three-dimensional orifices.

2. Typical two-dimensional flow nets, two-dimensional sluice gate, forces acting on a fluid mass, and uniform flow in pipes and open channels. 


\title{
RIVER HYDRAULICS
}

\section{SELECTED TOPICS OF FLUID MECHANICS}

\author{
By Cari E. Kindstater*
}

\section{ABSTRACT}

The fundamental equations of fluid mechanics are specific expressions of the principles of motion which are ascribed to Isaac Newton. Thus, the equations which form the framework of applied fluid mechanics or hydraulics are, in addition to the equation of continuity, the Newtonian equations of energy and momentum. These basic relationships are also the foundations of river hydraulics.

The fundamental equations are developed in this report with sufficient rigor to support critical examinations of their applicability to most problems met by hydraulic engineers of the Water Resources Division of the United States Geological Survey. Physical concepts are emphasized, and mathematical procedures are the simplest consistent with the specific requirements of the derivations. In lieu of numerical examples, analogies, and alternative procedures, this treatment stresses a brief methodical exposition of the essential principles.

An important objective of this report is to prepare the user to read the literature of the science. Thus, it begins with a basic vocabulary of technical symbols, terms, and concepts. Throughout, emphasis is placed on the language of modern fluid mechanics as it pertains to hydraulic engineering. The basic differential and integral equations of simple fluid motion are derived, and these equations are, in turn, used to describe the essential characteristics of hydrostatics and piezometry. The one-dimensional equations of continuity and motion are defined and are used to derive the general discharge equation. The flow net is described as a means of demonstrating significant characteristics of two-dimensional irrotational flow patterns. A typical flow net is examined in detail.

The influence of fluid viscosity is described as an obstacle to the derivation of general, integral equations of motion. It is observed that the part played by viscosity is one which is usually dependent on experimental evaluation. It follows that the dimensionless ratios known as the Euler, Froude, Reynolds, Weber, and Cauchy numbers are defined as essential tools for interpreting and using experimental data. The derivations of the energy and momentum equations are treated in detail. One-dimensional equations for steady nonuniform flow are developed, and the restrictions applicable to the equations are emphasized.

Conditions of uniform and gradually varied flow are discussed, and the origin of the Chezy equation is examined in relation to both the energy and the momentum equations. The inadequacy of all uniform-flow equations as a means of describing gradually varied flow is explained. Thus, one of the definitive problems of river hydraulics is analyzed in the light of present knowledge.

* Rogents-Professor of Oivil Engineering; Georgla Institute of Techmology, Atlanta, Ga.; consultant to the ర. S. Geological Survey. 


\section{INTRODUCTION}

This report is the outgrowth of a series of short schools conducted during the spring and summer of 1953 for engineers of the Surface Water Branch, Water Resources Division, U. S. Geological Survey. The topics considered are essentially the same as the topics selected for inclusion in the schools. However, in order that they might serve better as a guide and outline for informal study, the arrangement of the writer's original lecture notes has been considerably altered.

The purpose of the report, like the purpose of the schools which inspired it, is to build a simple but strong framework of the fundamentals of fluid mechanics. It is believed that this framework is capable of supporting a detailed analysis of most of the practical problems met by the engineers of the Geological Survey.

It is hoped that the least accomplishment of this work will be to inspire the reader with the confidence and desire to read more of the recent and current technical literature of modern fluid mechanics.

\section{TECHNICAL SYMBOLS, TERMS, AND BASIC CONCEPTS}

The material in this section is the basic language of fluid mechanics. The reader is urged to become thoroughly acquainted with these symbols, terms, and fundamental concepts before reading the subsequent text.

\section{LETTER SYMBOLS AND UNITS}

\section{BOUNDARY CONDITIONS}

$A$

$b, B$

$D$

$L$

$n$

$P$

$R$

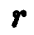

$\mathbf{s}$

$S$

Area; sq $\mathrm{ft}$ or $\mathrm{ft}^{2}$.

Width of section; $\mathrm{ft}$.

Diameter of a circular section; $\mathrm{ft}$.

A length, as defined; $\mathrm{ft}$.

Direction or distance perpendicular to streamlines in the $s, n$ plane; as a radial distance it is measured positively toward the center of curvature; $\mathrm{ft}$.

Wetted perimeter or perimeter of the wetted cross section; ft.

Hydraulic radius; ratio of the area to the wetted perimeter; ft.

Radius of a circular arc or radial distance; as a distance it is measured positively from the center of curvature; $\mathrm{ft}$.

Direction or distance along or parallel to streamlines; ft.

Hydraulic "slope"; in uniform-flow equations it is the flow-distance gradient of either the total energy head or the piezometric head. 
$\forall$
$m$
$x$
$y$
$z$

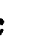

$\boldsymbol{y}$

$z$

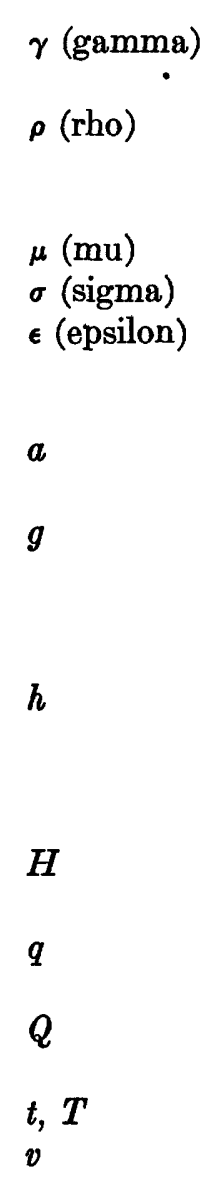

Volume; cu ft or $\mathrm{ft}^{3}$.

Direction or distance perpendicular to streamlines in the $s, m$ plane and at right angles to the $n$ direction; ft.

A direction or distance; as a plane coordinate it is usually horizontal; $\mathrm{ft}$.

A direction or distance; as a plane coordinate it is usually vertical; in open channels it is the depth of flow referred to the average bottom level; $\mathrm{ft}$.

Elevation of a point referred to a horizontal datum; ft.

\section{FLUID PROPERTIES}

Specific or unit weight; weight per unit volume; lb per cu $\mathrm{ft}$ or $\mathrm{lb} / \mathrm{ft}^{3}$.

Mass density; ma'ss per unit volume; also, ratio of specific weight to acceleration of gravity; slugs per cu $\mathrm{ft}$, slugs $/ \mathrm{ft}^{3}$, or $\mathrm{lb}-\mathrm{sec}^{2} / \mathrm{ft}^{4}$.

Viscosity; dynamic viscosity; lb-sec/ $\mathrm{ft}^{2}$.

Surface tension; lb/ft.

Modulus of elasticity; psf or $\mathrm{lb} / \mathrm{ft}^{2}$.

\section{MOTION (FLOW) PROPERTIES}

Acceleration; rate of change of velocity with respect to time; a vector quantity; $\mathrm{ft} / \mathrm{sec}^{2}$.

The acceleration due to gravitational force, usually taken to be essentially constant in magnitude (32.2) and fixed in direction (vertically downward); $\mathrm{ft} / \mathrm{sec}^{2}$.

Piezometric head; in general, equivalent to pressure head plus elevation; for uniform flow in open channels, it is equivalent to the elevation of the free surface; $\mathrm{ft}$.

Total energy head; energy in ft-lb/lb of fluid flowing; ft.

Volume rate of flow per unit width; discharge per foot of width; cfs per $\mathrm{ft}$ or $\mathrm{ft}^{3} / \mathrm{sec} / \mathrm{ft}$.

Volume rate of flow; total discharge at a cross section; $\mathrm{cfs}$ or $\mathrm{ft}^{3} / \mathrm{sec}$

An interval of time; sec.

Velocity at a point; instantaneous time rate of displacement of a particle or point; a vector quantity as distinguished from speed, which is scalar; fps or $\mathrm{ft} / \mathrm{sec}$. 


\section{V}

$F$

$M$

$p$

W

$\tau$ (tau)

$\eta$ (eta)

C

Co

$C_{\text {ch }}$

$C_{d}$

$C_{L}$

$C_{\tau}$

$K$

n

$\alpha$ (alpha)

$\beta$ (beta)

$\mathbf{E}$

$\mathbf{F}$
Average velocity in a cross section; ratio of total discharge to the normal area of a cross section; fps or $\mathrm{ft} / \mathrm{sec}$.

\section{FORCE PROPERTIES}

Force; a vector quantity; lb.

Mass; ratio of weight to the acceleration due to gravity; a scalar quantity; slugs.

Pressure intensity; normal (pressure) force per unit area; a scalar quantity; psf or lb/ft ${ }^{2}$.

Weight; total force due to gravity; a vector quantity, always vertical; lb.

Shear stress; tangential (shear) force per unit area; psf or $\mathrm{lb} / \mathrm{ft}^{2}$.

Eddy viscosity; apparent viscosity due to turbulence; lb-sec/ $\mathrm{ft}^{2}$.

RATIOS AND COFFFICIENTS

Any coefficient; usually dimensionless.

Coefficient of contraction; ratio of area of contracted stream to gross area of opening; dimensionless.

Coefficient in the Chezy equation; has dimensions of $\sqrt{g}$, units of $\mathrm{ft}^{1 / 2} / \mathrm{sec}$.

Coefficient of discharge; dimensionless.

Energy-loss coefficient; dimensionless.

Shear-force coefficient; dimensionless.

A constant.

Coefficient in the Chezy-Mànning equation; has dimensions of $R^{36}$, units of $\mathrm{ft}^{36}$.

Velocity-head coefficient; ratio of the true average velocity head in a cross section to the velocity head computed on the basis of the average velocity in the section; dimensionless.

Momentum coefficient; ratio of true rate of transfer of momentum (momentum flux) at a cross section to the momentum flux computed on the basis of the average velocity in the section; dimensionless.

Euler number; ratio of a unit inertial reaction to a unit pressure force; a basic flow parameter.

Froude number; ratio of a unit inertial reaction to a unit weight force; a number which varies inversely with the relative influence of weight on the flow pattern; dimensionless. 
$\mathbf{R}$

$\mathbf{W}$

C

$\Delta(\dot{d}$ elta $)$

$\Sigma$ (sigma)

$\propto$

$\approx$

$\rightarrow$

Reynolds number; ratio of a unit inertial reaction to a unit viscous shear force; a measure of the relative influence of viscosity; dimensionless.

Weber number; ratio of a unit inertial reaction to a unit surface-tension force; a measure of the relative influence of surface tension; dimensionless.

Cauchy number; ratio of a unit inertial reaction to a unit elastic force; a measure of the relative influence of elasticity; dimensionless.

\section{MATEEMATICAI SYMBOLS}

Change in value; final value minus initial value; increment.

Summation.

Proportional to; varies as.

Approximately equal.

Vector addition.

\section{BASIC CONCEPTS OF MECHANICS}

Newton's laws of motion.-Engineering mechanics, which includes the mechanics of solid and rigid bodies as well as fluids, is based on Newton's hypotheses or "laws" concerning the behavior of masses acted upon by external forces. The fundamental proposition is stated in the second law of motion: a mass acted upon by an unbalanced external force or force system has an acceleration which is proportional to and in the direction of the resultant force. This proposition is expressed algebraically in the familiar relation $\Sigma F \propto M a$, in which both $F$ and $a$ are recognized as vector quantities. In a rectilinear coordinate system, for example, $\Sigma F_{x} \propto M a_{x}, \Sigma F_{y} \propto M a_{y}$, and $\Sigma F_{2} \propto M a_{z}$.

The first law is: A mass remains at rest or moves with constant velocity in a straight line until acted upon by an unbalanced resultant external force; or, in accordance with the second law, if $\Sigma F=0$, then $a=0$. The first law, as applied to fluid mechanics, describes both hydrostatic and uniform-flow conditions.

The third law is: A mass acted upon by another mass exerts an equal, opposite, and simultaneous reaction. If $\Sigma F$ is defined as the resultant external force acting on a mass, then $M a$ is the inertial reaction of the mass.

It is apparent that Newton's second law of motion provides a quantitative expression for the relationship between force, mass, and acceleration which is the basis for all equations of motion in engineering mechanics. 
Dimensions.-The dimension of a quantity is its classification according to a system of independent physical categories. Because Newton's second law is a complete description of a mass in motion, the fundamental dimensions are the dimensions of the quantities involved in a physical statement of the law. Thus, the fundamental dimensions are mass, length, time, and force $(M, L, T$, and $F$ ) from the relationship $F \propto M a \propto M L / T^{2}$. Quantities such as acceleration, power, and momentum are derived quantities; their dimensions are expressed in terms of the fundamental dimensions. Because the 4 fundamental dimensions are related by means of the Newtonian equation, an adequate dimensional system need involve only 3 dimensions. Commonly used dimensional systems involve $F, L$, and $T$, or $M, L$, and $T$.

Units of measure.-For each of the fundamental dimensions there is an accepted unit of measure. Thus, $L$ is measured in feet, $F$ in pounds, $T$ in seconds, and $M$ in slugs. Only three units need be involved in a complete dimensional system, however. For example, slugs need not be used in the $F, L, T$ system. The units of derived quantities such as acceleration, power, and momentum are expressed only in terms of the units of the fundamental dimensions.

The American engineering system of units is an absolute system; that is, in the basic relationship $F \propto\left(M L / T^{2}\right)=K\left(M L / T^{2}\right)$, the proportionality constant, $K$, is taken to be unity. Thus, a force of 1 pound produces in a mass of 1 slug an acceleration of 1 foot per second per second. It follows that, expressed in the absolute system of units, Newton's second law can be written $\Sigma F=M a$.

Kinematics.-Kinematics deals with the description of motion without respect to the forces which cause or influence this motion. Kinematics involves only time and space.

Dynamics.-Dynamics deals with the effect of forces in causing or changing the motion of a mass. It is further subdivided into statics and kinetics.

Statics.-Motions described under this heading correspond to the condition of zero acceleration in the directions considered.

Kinetics.-Kinetics is that branch of dynamics which deals with the accelerated motion of a mass under the influence of unbalanced external forces.

Work.-Work is the product of a force and the distance over which the force acts in accomplishing motion. Work is a scalar quantity; it does not involve the time during which the force acts. Work is measured in foot-pounds ( $\mathrm{ft}-\mathrm{lb}$ ).

Power.-Power is the time rate of doing work. The power delivered to a moving body is proportional to the product of the foree producing the motion and the velocity of the body in the direction of 
the motion. Power is a scalar quantity. The unit of power is footpounds per second ( $\mathrm{ft}-\mathrm{lb}$ per sec) or horsepower. One horsepower is equivalent to $550 \mathrm{ft}-\mathrm{lb}$ per sec.

Energy.-A body upon which work is accomplished acquires thereby the capacity to do an equal amount of work. The capacity to do work is called energy. Thus, energy is a measure of work, and vice versa. Energy is a scalar quantity. The unit of both work and energy is the foot-pound ( $\mathrm{ft}-\mathrm{lb}$ ).

Momentum.- - Linear momentum is the product of mass and velocity. It is a vector quantity, and its unit is the pound-second (lb-sec).

Impulse.-Linear impulse is the product of a force and the time during which the force acts. It is a vector quantity. From Newton's second law, impulse accomplishes and is equivalent to the change in momentum experienced by the mass asted upon. The unit of impulse is, therefore, the same as that of momentum, the pound-second (lb-sec).

\section{BASIC CONCEPTS OF FLUID MECHANICS}

Continuity and discharge.-Fluid mass can be neither created nodestroyed by virtue of its motion. Thus, in a steady fluid flow ber tween solid boundaries or through a streamtube, the rate of passage of fluid mass through successive cross sections is constant. If the fluid be one of negligible compressibility (constant density), the rate of passage of fluid volume past successive sections is also constant. This is the simple basis for the continuity principle. The principle is expressed, for example, in the equation $Q=A V=$ constant, where $-Q$ is the volume rate of flow, or discharge, in cubic feet per second and $V$ is the average velocity normal to the surface represented by the area $(A)$. In a section of nonuniform velocity, the total discharge is the summation (or integral) of the discharges through the elementary areas comprising the total area of the section.

Uniform and steady motion.-Velocity in a fluid motion is a vector quantity and it is a function of both time and space. When the velocity remains constant with respect to time, the flow is described as steady. Most problems in engineering hydraulics involve steady motion.

When the velocity is constant with respect to distance in the direction of motion, the flow is described as uniform. Uniform flow occurs in long pipes. It seldom occurs in natural open channels.

Rotational and irrotational motion.-The distinction between rotational and irrotational motion is essentially mathematical. The most significant characteristics of an irrotational flow are those usually associated with the classical "ideal" or "frictionless" fluid (a fluid without compressibility or viscosity). It is pertinent to the subse- 
quent use of the flow net in these notes to observe that irrotationality is corequisite to the existence of a flow net.

Laminar and turbulent motion.-Laminar fluid motion is characterized by a steady, translatory movement of all particles (small elements) of the fluid. Turbulence imposed on a laminar flow is eventually damped out by the viscous shear forces. Because viscous forces are large relative to inertial reactions in laminar motion, this condition corresponds to low values of the Reynolds number.

Turbulent motion is characterized by an erratic, small-scale eddying motion which is superposed on the translatory movement of the fluid mass. Local disturbances are rapidly dispersed throughout a turbulent fluid flow because the viscous shear forces are small relative to the inertial reactions resulting from the turbulence. Thus, turbulent flow corresponds to high values of the Reynolds number. For most practical purposes, motion which is turbulent is described in terms of the average translatory velocity at a point or in a cross section.

Critical, tranquil, and rapid motion.-If the mean total energy head referred to the bottom of an open channel is a minimum for a given discharge, the flow is described as critical. When critical flow occurs in a straight, rectangular, or nearly rectangular channel in which the velocity in the cross section is essentially constant, the average velocity is theoretically equal to the velocity of a small gravity wave, and the Froude number is equal to unity. The flow is described as tranquil if the average velocity is less than the critical value. If the average velocity is greater than the critical value, the flow is described as rapid.

Shear and pressure.-The total force on any surface within a fluid in motion can be resolved into tangential and normal components. The tangential component of the total force on the surface is the shear force $\left(F_{\tau}\right)$. The limit of the ratio of the shear force to the area of the surface as the area approaches zero is the shear stress at a point $(\tau)$.

The normal component of the total force on an area is the pressure force $\left(F_{p}\right)$. The limit of the ratio of the pressure force to the area as the area approaches zero is the pressure intensity $(p)$ at a point. Pressure intensity is a scalar quantity.

A fluid at rest with respect to its boundaries is in a state of zero shear stress throughout. It follows that pressure changes imposed at one point in a fluid mass at rest are transmitted undiminished to all points in the fluid mass (Pascal's principle). Furthermore, because shear stresses cannot exist in a fluid at rest, the resultant force on any surface within the fluid is a pressure force, and it is normal to the surface.

Viscous and turbulent shear.-The viscous-shear force $\left(F_{\mu}\right)$ is a consequence of internal stresses which resist fluid deformation. Thus, 
relative movement of the fluid on adjacent stream surfaces in a laminar flow results in a viscous-shear stress $\left(\tau_{\mu}\right)$. In a motion of this kind, $\tau_{\mu}$ is a function of the fluid viscosity and the velocity "gradient", a property of the flow defined as the rate of change of the tangential velocity with respect to distance perpendicular to the motion. The viscousshear stress at any point in the flow pattern, therefore, is $\tau_{\mu}=$ $\mu\left(d v_{s} / d n\right)$, where $\mu$ is the dynamic viscosity of the fluid and $d v_{s} / d n$ is the velocity gradient at the point. It is significant that $\tau_{\mu}$ is independent of fluid density and pressure.

The total shear force $\left(F_{\tau}\right)$ acting on a fluid in turbulent motion is the sum of the viscous shear $\left(F_{\mu}\right)$ and the turbulent shear $\left(F_{\eta}\right)$. Turbulent shear is a consequence of the momentum exchange between small masses of fiuid moved transversely by the action of the turbulence eddies. Unlike viscous-shear stress, turbulent-shear stress $\left(\tau_{\eta}\right)$ is a function of fluid density, and it is independent of fluid viscosity:

It can be shown that the turbulent-shear force acting on a fluid element is directly proportional to its inertial reaction. It follows that the Reynolds number is proportional to the ratio of turbulent shear to viscous shear, or $\mathbf{R} \propto \tau_{\eta} / \tau_{\mu}$. The influence of viscous shear is negligible in fully developed turbulence.

Boundary layer.-The boundary layer was originally conceived as a means of correlating real fluid motion with the theoretical behavior of the hydrodynamicist's ideal fluid. Thus, Prandtl suggested that in flows at high Reynolds numbers the effect of fluid viscosity could be assumed to be limited to a narrow region near the confining boundaries. This concept is of fundamental importance in the analysis of flow around bodies immersed in relatively large fluid spaces. It is also essential to an understanding of certain phenomena associated with the establishment of uniform flow at entrances to conduits or following local disturbances in an otherwise uniform conduit. In regions of fully established flow, as in long pipes and open channels, the boundary layer is virtually the entire cross section of the flow.

Streamlines and streamtubes.-A streamline is a line drawn through a flow in such a manner that at every point along its length it is tangent. to the velocity vector. It follows that there can be no flow across a streamline; in this sense, the streamline imposes a boundary condition on the adjacent flow.

A streamtube is a surface formed entirely of streamlines; in other words, it is the surface defined by the streamlines passing through a closed curve. As the boundary of a streamtube is comprised of streamlines, there can be no velocity and, therefore, no flow through the walls of a streamtube. Any solid boundary or separation surface: which effectively guides the flow has the properties of a streamtube surface. 
Stagnation and separation.-A stagnation point in fluid motion is defined as a point of zero velocity. When boundary conditions cause a flow to undergo an abrupt convergence, a tendency for stagnation occurs at a point on the boundary. In a sharp, concave boundary corner, or on the nose of a blunt obstruction, for example, the flow along the boundary would apparently experience an instantaneous change in direction of motion. As this implies an infinite normal acceleration if the tangential velocity were anything but zero, stagnation occurs.

When flow boundaries diverge abruptly, as in sudden enlargements or following blunt obstructions, the boundary ceases to guide the flow effectively and separation occurs. Thus, the apparent requirement that the fluid undergo an infinite acceleration at the boundary discontinuity does not, in this example, result in stagnation. Instead, the live stream separates from the boundary and follows a "freestream" surface bounded by eddying fluid. As a consequence of viscosity, such eddy-filled separation zones also occur in corners near regions of boundary convergence, thereby preventing stagnation.

\section{EQUATIONS OF MOTION}

\section{FUNDAMENTAI EQUATION OF MOTION}

The principal objective of fluid mechanics is a mathematical description of fluid motion. Newton's second law is the basis for equations of fluid motion. The sense of Newton's proposition is contained in the algebraic expression ${ }^{1} \Sigma F \propto M a=K M a$. This is a vector relationship which states that the resultant of all external forces acting in a particular direction on a mass is proportional to the product of the mass and its acceleration in the direction of the resultant force. If the quantities involved in this expression are evaluated in accordance with an absolute system of units, then $K=1.0$, or $\Sigma F=M a$. In the Cartesian space system, for example, $\Sigma F_{x}=M a_{x}$, $\Sigma F_{y}=M a_{y}$, and $\Sigma F_{z}=M a_{z}$.

\section{EXTERNAL FORCES IN FLUID MOTION}

The independent forces which influence fluid motion are the consequence of 1 property of state (pressure) and 4 physical properties of the fluid (density, viscosity, surface tension, and elasticity). An additional force, the apparent shear force due to fluid turbulence $\left(F_{\eta}\right)$, is not recognized as an independent force because it is directly proportional to the inertial characteristics of the flow.

Pressure, or pressure intensity, is a scalar quantity which describes

\footnotetext{
1 Except where emphasis is intended, the symbols and technical terms defined in the preceding section are used in the subsequent text without further explanation.
} 
a particular kind of fluid stress. Pressure is the cause of a resultant external force $\left(F_{p}\right)$ only when it varies in the direction of motion.

Density is involved in fluid motion in two distinctly different ways. Thus, density accounts for an accelerative force $\left(F_{g}\right)$ which is the component of the weight $(W)$ in the direction considered. The definitive equation, $W=M g=\rho \forall g$, is a particular form of Newton's second law in which $\rho$ is the density and $g$ is described as the acceleration due to gravity. On the other hand, the total inertial reaction due to the resultant of all the external forces, which might or might not include a component of the weight force, also involves the density; that is, $\Sigma F=M a=\rho \forall a$.

Viscosity is a fluid property which accounts for a shear force $\left(F_{\mu}\right)$ whenever fluids are in motion with respect to their boundaries. Viscous shear is the result of molecular forces which oppose fluid deformation. In turbulent fluid motion the total shear force $\left(F_{\tau}\right)$ is the sum of $F_{\mu}$ and the turbulent shear force $\left(F_{\eta}\right)$.

Surface tension is the name given to certain manifestations of molecular forces which occur at the interface between different substances; for example, between a free jet of water and the surrounding air. The surface-tension force $\left(F_{\sigma}\right)$ is ordinarily of appreciable magnitude only when the other forces involved are extremely and unusually small.

Elasticity, or forces due to elastic deformation $\left(F_{\epsilon}\right)$, are involved primarily in the unsteady flow of liquids and in the flow of gases under high pressure gradients. Neither of these problems is pertinent to this discussion.

\section{KINEMATICS OF FIUID MOTION}

A mathematical description of fluid motion necessarily involves the space-time characteristics which are known as velocity and acceleration.

Velocity is the time rate of displacement of a mass or a point. For example, if $\Delta s$ is a small displacement or distance traversed along a streamline in a short time interval $(\Delta t)$, and if $v_{s}$ is the average tangential velocity in the interval on that part of the streamline, then $v_{s}=\Delta s / \Delta t$. It follows that the instantaneous tangential veloeity at a point on a streamline is $v_{s}=d s / d t$.

Velocity is a vector quantity. Thus, the total or resultant velocity at a point is the vector sum of its components on the various vector axes. From the definition of a streamline, the total velocity in the "natural" or $s, n, m$ system of coordinates is $v_{s}$, and the normal components, $v_{n}$ and $v_{m}$, are zero. For many purposes, however, the Cartesian or $x, y, z$ system of describing motion in three-dimensional space is preferred. Thus, in the Cartesian system $v=v_{x} \nmid v_{y} \rightarrow v_{z}=$ 
$\sqrt{v_{x}{ }^{2}+v_{y}{ }^{2}+v_{z}{ }^{2}}$, where $\rightarrow$ indicates vector addition and $v_{x}=d x / d t$, $v_{y}=d y / d t$, and $v_{z}=d z / d t$.

Acceleration is time rate of change of velocity. As this discussion is concerned with steady motion only, the variation of the velocity at a point, with respect to time, is always zero. Thus, in the general definition $a=d v / d t$, the change in velocity represented by $d v$ is the change which occurs over the, distance traversed during the time interval $d t$. It follows that the total acceleration in steady motion is described as convective acceleration.

In the natural system of coordinates, the total acceleration includes both tangential and normal components. Thus, $a_{s}=d v_{s} / d t$ is the tangential acceleration. Similarly, $a_{n}=d v_{n} / d t$ and $a_{m}=d v_{m} / d t$ are the orthogonal components of the normal acceleration. In the Cartesian system the total convective acceleration is comprised of the components $a_{x}=d v_{x} / d t, a_{y}=d v_{y} / d t$, and $a_{z}=d v_{z} / d t$.

For subsequent use, the tangential and normal accelerations in two-dimensional motion $\left(a_{m}=0\right)$ are more conveniently expressed

$$
a_{s}=\frac{d v_{s}}{d t} \frac{d s}{d s}=\frac{d v_{s}}{d s} \frac{d s}{d t}=v_{s} \frac{d v_{s}}{d s}
$$

or, from the calculus,

$$
a_{s}=v_{s} \frac{d v_{s}}{d s}=\frac{1}{2} \frac{d v_{s}^{2}}{d s}
$$

From any mechanics or physics text, the normal (centripetal) acceleration at a point on a streamline is

$$
a_{n}=\frac{d v_{n}}{d t}=\frac{d v_{n}}{d s} \frac{d s}{d t}=v_{s} \frac{d v_{n}}{d s}=\frac{v_{s}{ }^{2}}{r},
$$

in which $r$ is the radius of curvature of the streamline at the point.

It is significant that equation 1 describes $a_{s}$ as a measure of the rate of change of the magnitude of $v_{s}$, whereas equation 2 describes $a_{n}$ as a measure of the change in direction of $v_{s}$. Equation 2 also explains the anomalous situation which appears to exist when it is observed that $a_{n}$ is not necessarily equal to zero even though $v_{n}$ is always zero on a streamline.

\section{RQUATIONS FOR A SIMPLE FLUID MOTION}

The next logical step in writing an equation of fluid motion is to evaluate all of the forces acting on a typical accelerated fluid mass and to write an equation of the form

$$
\Sigma F=F_{p} \nmid \rightarrow F_{g}+F_{\tau}+F_{\sigma} \nmid \rightarrow F_{\varepsilon}=M a
$$


in each of the coordinate directions. First, however, in view of the purpose of this discussion, the problem can be simplified by omitting further consideration of the relatively minor surface-tension and elastic forces, $F_{\sigma}$ and $F_{\epsilon}$, respectively. Furthermore, in order to demonstrate certain basic principles without the complication of the most troublesome of all forces-those due to viscosity and turbulencea kind of motion in which the influence of $F_{r}$ is negligible is initially selected for analysis.

The flow of a fluid from a large quiescent reservoir through a small orifice is shown in plate $1(\mathrm{a}-\mathrm{c})$. It is assumed that the orifice discharges into another reservoir containing a fluid of the same density in order that certain independent influences of fluid weight can also be ignored. Sufficient.generality and considerable convenience is achieved in this example by assuming that the flow is two-dimensional in the $s, n$ plane. Thus, the only accelerations involved are $a_{s}$ and $a_{n}$, and the only forces involved are those which have components acting parallel and perpendicular to the streamlines. The natural system of coordinates is ideally suited to this analysis.

Plate 1 (b) shows a typical small mass of the fluid in motion. For convenience it is shown as a prismatic element of volume $(\Delta s \Delta A)$. Its axis is a streamline and it is as small as necessary in order that its motion can be described in terms of the motion of its mass center. In plate 1 (c), showing the same element as a free body, the pressure force on each end of the element is represented by the product of the average pressure and the end area. The weight force in the direction of motion $\left(F_{g}\right)$ is shown as the $s$ component of the total weight of the element $(W)$. It should be apparent that any errors resulting from neglecting area, velocity, or pressure variations over the small volume $(\Delta s \Delta A)$ are of a second order of magnitude and that they disappear when the volume of the element is allowed to approach the infinitesi$\operatorname{mal}(d s d A)$.

In the kind of motion represented by plate $1(\mathrm{a}-\mathrm{c})$ the influence of the total shear force $\left(F_{\tau}\right)$ is negligible. This follows from the fact that motion in the reservoir is assumed to be nonturbulent and that the velocity of the fluid relative to the boundaries is negligible except immediately adjacent to the opening. Thus, neither viscous- nor turbulent-shear forces are appreciable. Flow patterns of this kind are essentially similar to the theoretical flow patterns described in hydrodynamics for an ideal fluid. Thus, for the motion pattern described,

$$
\Sigma F_{s}=F_{p}+\rightarrow F_{\mathrm{g}}=M a_{s},
$$

in which $F_{p}$ and $F_{g}$ are the $s$ components of the forces due to pressure and weight, respectively. 
From plate 1 (c), the resultant pressure force is

$$
F_{p}=p \Delta \dot{A}-(p+\Delta p) \Delta A=-\Delta p \Delta A .
$$

If $i t$ is assumed that the pressure intensity increases at a uniform rate $(d p / d s)$ in the direction of motion, then $\Delta p=(d p / d s) \Delta s$ is the total change in pressure over the distance $\Delta s$. Substituting and simplifying,

$$
F_{p}=-\frac{d p}{d s}(\Delta s \Delta A)
$$

The weight of the element is $\gamma(\Delta s \Delta A)$. The $s$ component of the weight is, from plate 1 (c),

$$
F_{\mathrm{g}}=-\gamma(\Delta s \Delta A) \sin \theta=-\gamma(\Delta s \Delta A) \frac{d z}{d s} .
$$

The mass of the element is $\rho(\Delta s \Delta A)$, and the inertial reaction due to $F_{p}$ and $F_{z}$ is

$$
M a_{s}=\rho(\Delta s \Delta A) a_{s} .
$$

Combining equations 5,6 , and 7 with equation 4 ,

$$
-\frac{d p}{d s}(\Delta s \Delta A)-\gamma \frac{d z}{d s}(\Delta s \Delta A)=\rho a_{s}(\Delta s \Delta A) .
$$

Reducing this to a force-per-unit-volume relationship by dividing by the volume $(\Delta s \Delta A)$ of the element,

$$
-\frac{d p}{d s}-\gamma \frac{d z}{d s}=-\frac{d}{d s}(p+\gamma z)=\rho a_{s} .
$$

This equation shows that acceleration in the direction of motion results only when there is a gradient (a change with respect to distance) of the sum $p+\gamma z$ in that direction. It shows also that when $d z / d s=0$, which is true of any motion in a horizontal plane, the pressure gradient $(-d p / d s)$ is the sole accelerative force per unit volume, and it is exactly equal to the inertial reaction per unit volume $\left(\rho a_{s}\right)$. It indicates furthermore that when $d p / d s=0$, then $-\gamma(d z / d s)=\rho a_{s}$, or $-d z / d s=a_{s} / g$, equations which, for example, describe flow along any free (constant-pressure) surface; thus, when $d s=-d z$, as in free fall, $a_{s}=g$, or the total acceleration is equal to the acceleration due to gravity. Finally, it indicates that when $a_{s}=0$, the pressure gradient is $d p / d s=-\gamma(d z / d s)$, or, in the distance $d s, d p=-\gamma d z$; this describes the condition of hydrostatic pressure distribution. 
If $a_{s}=\frac{1}{2} \frac{d v_{s}{ }^{2}}{d s}$ from equation 1 is substituted in equation 9 and the terms rearranged,

$$
\frac{d}{d s}\left(p+\gamma z+\frac{\rho v_{s}{ }^{2}}{2}\right)=0
$$

From this equation, the $s$ rate of change of the quantity within parentheses, at any point on the streamline, is zero.

If equation 10 is multiplied by the differential distance $(d s)$,

$$
d\left(p+\gamma z+\frac{\rho v_{s}^{2}}{2}\right)=0,
$$

which shows that the differential change in the parenthetical quantity over the differential distance $(d s)$ is also zero. It follows that the quantity within the parentheses is a constant along the streamline. Thus,

$$
p+\gamma z+\frac{\rho v_{s}^{2}}{2}=\mathrm{K}
$$

which is the integral of equation 11 , is a relationship between pressure, elevation, and velocity in which $K$ is the constant of integration for a particular streamline. At successive points along this line, therefore,

$$
\frac{\rho v_{s_{1}}^{2}}{2}+p_{1}+\gamma z_{1}=\frac{\rho v_{s_{2}}^{2}}{2}+p_{2}+\gamma z_{2}, \text { etc. }
$$

Equation 13 can be written in an alternate "head" form, known more familiarly to civil-hydraulic engineers as the Bernoulli equation. Dividing by the constant specific weight of the liquid $(\gamma)$ and noting that $\gamma / \rho=g$,

$$
\frac{v_{s_{1}}^{2}}{2 g}+\frac{p_{1}}{\gamma}+z_{1}=\frac{v_{s_{2}}^{2}}{2 g}+\frac{p_{2}}{\gamma}+z_{2}
$$

The algebraic operations which led from the Newtonian equation to equation 13 involved dividing a force by a volume and multiplying by a distance. Thus, the units of every term in the equation are foot-pounds per cubic foot. It follows that the units of every term in equation 14 are foot-pounds per pound or, simply, feet.

Equations 13 and 14 are both properly described as Bernoulli equations. They are relationships between integral values of pressure, elevation, and velocity at successive points on a single streamline. In order to develop similar relationships between points on adjacent streamlines it is necessary that the foregoing analysis be repeated with the axis of the definitive fluid element oriented in the 
$n$ direction. As a result, a differential equation is obtained which is the counterpart of equation 9 :

$$
-\frac{d}{d n}(p+\gamma z)=\rho a_{n}
$$

However, when the equivalent expression for $a_{n}$ in terms of the velocity $\left(v_{s}\right)$ is substituted, the similarity in the two developments ceases. Thus, $a_{n}=v_{s}{ }^{2} / r$ from equation 2 . When this is substituted in equation 15, it appears to yield a nonintegrable equation. Returning to equation 2, however, let $a_{n}=v_{s}\left(d v_{n} / d s\right)$. Adding and subtracting the quantity $v_{s}\left(d v_{s} / d n\right)$,

$$
a_{n}=v_{s} \frac{d v_{n}}{d s}+v_{s} \frac{d v_{s}}{d n}-v_{s} \frac{d v_{s}}{d n}=v_{s} \frac{d v_{s}}{d n}+v_{s}\left(\frac{d v_{n}}{d s}-\frac{d v_{s}}{d n}\right) .
$$

From hydrodynamics, the quantity within the parentheses in the last term is a measure of rotationality. Irrotational flow corresponds to the condition that $\left(d v_{n} / d s\right)-\left(d v_{s} / d n\right)=0$. Whereas this distinction between rotational and irrotational flow is essentially mathematical, it is significant that hydrodynamics also shows that the absence of viscous shear is corequisite to irrotationality. Thus, because shear forces are negligible here, it can be assumed that the flow is irrotational, and, therefore,

$$
a_{n}=v_{s} \frac{d v_{s}}{d n}=\frac{1}{2} \frac{d v_{s}^{2}}{d n}
$$

When this is substituted in equation 15 , the modified equation leads to differential relationships which are the counterparts of equations 10 and 11 and integral relationships which are identical with the Bernoulli equations $(12,13$, and 14). Thus, when the shear forces are negligible, the same relationships between velocity, pressure, and elevation will apply between adjacent streamlines as apply along single streamlines. In other words, the constant of integration in equation 12 is the same for all streamlines and the Bernoulli equation is applicable to all points in an irrotational or frictionless flow pattern.

Several important applications of the equations of motion, including hydrostatics, piezometry, the one-dimensional equation, and the discharge equation, are discussed in the following sections.

\section{HYDROSTATICS}

When only pressure and weight forces are effective in a fluid motion, as in the preceding example, the condition described as hydrostatic pressure distribution occurs along any line on which the acceleration at all points is zero. Thus, with $a_{s}=0$ in equation 9 , hydrostatic 
pressure distribution in the $s$ direction implies that the pressure in an incompressible fluid ( $\gamma$ is constant) varies inversely with the elevation along a streamline. Similarly, from equation 15, pressure distribution is hydrostatic along lines normal to the streamlines when $a_{n}=0$. This also means, of course, that pressure is not hydrostatically distributed in the $n$ direction if the streamlines are curved. It follows that hydrostatic pressure distribution occurs throughout a fluid in motion only if shear forces are negligible and the flow is truly uniform; that is, if $a_{s}=0$ and $a_{n}=0$.

However, when the shear force $\left(F_{\tau}\right)$ cannot be ignored, it should be apparent from plate 1 (c) that the pressure must vary with the influence of this force as well as with elevation along the streamline. Thus, in general, pressures are hydrostatically distributed in the $s$ direction of a uniform motion only when the relative velocity of fluid and boundary is so small that the shear force is negligible. On the other hand, pressures are hydrostatically distributed in the $n$ direction as long as $a_{n}=0$. This follows from the fact that, from the definition of a streamline, the average normal velocity $\left(v_{n}\right)$ is zero and, as a consequence, the net shear force in the $n$ direction is always zero.

Hydrostatics, as the term is generally used in technical literature, refers to the behavior of fluids, particularly liquids, at rest. The equations derived for a simple fluid motion are also applicable to hydrostatics. For this application, as there is no flow and consequently there are no streamlines, the $s$ direction is arbitrarily defined as any direction. From equation 9 , when the fluid is at rest the acceleration in all directions is zero, and the equation

$$
-\frac{d p}{d s}=\gamma \frac{d z}{d s}
$$

defines the condition of hydrostatic pressure distribution throughout the fluid. Thus, along any line, in a distance $d s$,

$$
d p=-\gamma d z .
$$

In horizontal planes, $d z=0$, whence, from equation $18, d p=0$. Therefore, one of the basic principles of hydrostatics is that the pressure is the same at all points on horizontal planes in a fluid at rest.

When $\gamma$ is constant, which is sensibly true for liquids, integration of equation 18 yields

$$
p_{2}-p_{1}=\gamma\left(z_{1}-z_{2}\right) .
$$

Equation 19 is apparently a particular form of the Bernoulli equation (13). It is also the basic gage-pressure equation if conditions at point 1 are defined as the conditions on a liquid surface open to the atmos- 
phere. Thus, if $p_{1}=0$ (gage) and if $z_{1}-z_{2}=y_{2}$ is the vertical depth of point 2 below the free surface, then $p_{2}=\gamma y_{2}$. It follows that the gage pressure at any point in a continuous body of liquid at rest is

$$
p=\gamma y,
$$

in which $y$ is the vertical depth of the point below the real or imaginary free surface of the liquid.

\section{PIEZOMETR $Y$}

A piezometer is a small hole located in a surface which comprises a fluid boundary. The purpose of the piezometer is to provide a means of measuring the fluid pressure at the point where the hole is located. In its definitive application to a liquid, as shown in plate 1 (d), the piezometer is connected to an open tube called a manometer. When the pressure in the liquid at the piezometer is exactly equal to the pressure due to the weight of the liquid in the manometer column, the liquid in the manometer is at rest. Thus, the pressure at the point of attachment can be computed from the gage-pressure relationship (eq 20). When a piezometer is used to measure the pressure on the boundary of a flowing fluid, the piezometer orifice must be small and very carefully made in order that flow along the boundary is not disturbed by the presence of the piezometer.

The level of the liquid in a simple open-tube manometer is appropriately called the piezometric level. As its height above the level of the piezometer $(y)$ is equal to $p / \gamma$ from equation 20 , this distance is described as the pressure head at the point of attachment. The elevation of the point with respect to a horizontal datum is called its elevation head. Thus, the vertical distance between the piezometric level in the manometer and the elevation datum is described as the piezometric head at the point where the piezometer is located. From this definition, if the symbol $h$ is used to denote the piezometric head at any point,

$$
h=\frac{p}{\gamma}+z .
$$

Thus, from equation 15 (divided by $\gamma$ ),

$$
-\frac{d}{d n}\left(\frac{p}{\gamma}+z\right)=-\frac{d h}{d n}=\frac{\rho}{\gamma} a_{n}=\frac{a_{n}}{g}
$$

This equation shows that the piezometric-head distribution in a section normal to the flow is proportional to the normal acceleration. The hydrostatic condition in the $n$ direction occurs when $a_{n}=0$. For this condition the gradient of the piezometric head is zero; that is, the piezometric head is constant. Thus, when flow past $\varepsilon$ piezometer 
in a flow boundary is truly uniform, the piezometer indicates not only the piezometric head at the point of attachment but also the piezometric head at all points in the adjacent uniform flow. Under these circumstances, the piezometric level is independent of the elevation of the piezometer. These conclusions are illustrated in plate $1(d)$.

When a liquid flows in an open channel, as in plate 1 (f), the upper boundary of the flow is both a stream surface and a piezometric surface. The gage pressure over the upper stream surface, regardless of its configuration or velocity, is zero. Thus, a piezometer located on the boundary of a uniform flow in an open channel indicates not only the piezometric level but also the level of the flowing liquid at the point of attachment. It follows that, if the piezometric datum is defined as the bottom of the channel, a piezometer located anywhere in the cross section indicates the depth of the liquid in the channel at that section.

When a piezometer is located on the upstream end of a small object placed in a fluid flow, stagnation occurs over the piezometer. As viscosity has little influence on this occurrence, the Bernoulli equation is applicable between the stagnation point and any point on the same streamline in the undisturbed flow upstream from the obstruction. Two such points (on streamline $\mathrm{AB}$ ) in a uniform pipe flow are designated points 1 and 2 in plate 1 (e). If $v_{2}=0$, and $h=(p / \gamma)+z$ is substituted in equation 13 , the piezometric head at the stagnation point is

$$
h_{2}=h_{1}+\frac{v_{1}^{2}}{2 g} \text {. }
$$

In plate $1(\mathrm{e})$, the piezometer at point 2 is connected to an open manometer tube. The stagnation pressure on the nose of the tube causes liquid in the tube to rise to a height equal to $p_{2} / \gamma$ above the level of the piezometer or $h_{2}$ above the elevation datum. If the flow in the pipe is uniform at this section, a second piezometer located at any point on the boundary indicates the piezometric head in the undisturbed flow at point 1 . Thus, from equation 23 , the velocity head at point 1 is indicated as the difference in the piezometric levels in the two manometers. It follows that the velocity in the undisturbed flow on streamline $\mathrm{AB}$ is

$$
v_{1}=\sqrt{2 g\left(h_{2}-h_{1}\right)} .
$$

A tube such as that in plate 1 (e) which indicates the stagnationpressure head is called a stagnation or pitot tube. It is apparent that the pitot tube provides an accurate means of measuring velocities in fluid motion. Its application to open-channel flows is illustrated in plate $1(\mathrm{~g})$. 
The velocity in a normal section through a uniform fluid flow between fixed boundaries varies with distance from the boundary. This is incidentally a consequence of the shear stresses which result from the relative motion of a viscous fluid and a solid boundary. When a pitot tube is used to traverse such a section, the piezometric level indicated by the tube varies from one streamline to the next. However, the piezometric level indicated by all wall piezometers is the same as long as $a_{n}=0$ at the section. Thus, only the pitot-tube manometer indications are affected by the velocity variations in the section. These conclusions are illustrated for an open channel in plate $1(\mathrm{~h})$.

The equations of motion have been used to demonstrate some of the basic principles of piezometry in uniform fluid motion. These equations are equally valuable as aids in the interpretation of piezometric measurements in nonuniform flows. For example, piezometers are often located at various points on bridge piers or abutments as a means of measuring the average water-surface level in the bridge opening. If a piezometer is located downstream from the disturbed flow near the entrance to the constricted waterway, and if the flow across the entire section normal to the piezometer station is essentially parallel to the face of the pier or abutment on which the piezometer is located, the measurement will be quite satisfactory as an indication of the average water-surface level at this section.

Piezometers located near the upstream end of the structure, however, are generally unsatisfactory. Thus, piezometers located on the upstream face of a pier are ordinarily in the vicinity of a stagnation zone, and measurements are influenced to an indeterminate degree by separation upstream from the pier nose. The so-called superelevation of the water curving around the nose of a pier is evidence of nonhydrostatic pressure distribution due to normal acceleration in the horizontal plane. Similarly, vertical accelerations in this zone account for nonhydrostatic pressure distributions in vertical planes. Therefore, piezometers located on the walls of a pier just downstream from the nose indicate neither the mean watersurface level in the cross section nor the level adjacent to the pier.

It should be apparent that short piers, circular piers, piers with irregular boundaries, webbed pile bents, or piers alined obliquely with the flow are unsatisfactory as locations for stage-recorder gage intakes unless they are calibrated in the field or in the laboratory.

\section{ONE-DIMENSIONAL FQUATION}

The piezometric head has been shown to be constant in any normal section over. which $a_{n}$ is everywhere zero (eq 22). From equation 16, which applies only when the shear forces are negligible, the normal 
acceleration is proportional to the $n$ gradient of the tangential velocity. Thus, if $a_{n}=0$, it follows that $d v_{s} / d n=0$, or $v_{s}=v$ is a constant. It may be concluded, therefore, that at all points over a normal section both the tangential velocity and the piezometric head are constant when $F_{\tau}$ and $a_{n}$ are negligible. Two sections which satisfy these conditions are shown in plate 1 (i).

Plate 1 (i), like plate 1 (a), represents the flow of a fluid from a large reservoir through a small orifice into another reservoir. Section 1 in the figure is a hemispherical surface which is concentric with the center of the orifice. Streamlines passing through this surface are radial lines; therefore they satisfy the requirement that the streamlines be without curvature if $a_{n}$ be zero. As the influence of the shear forces is negligible in this kind of flow pattern, it is concluded that the piezometric head $\left(h_{1}\right)$ and the tangential velocity $\left(v_{1}\right)$ are constant at all points on section 1 . Section 2 is a section through the parallel boundaries of the jet, downstream from the nonuniform flow in the immediate vicinity of the orifice. As the streamlines in this section are straight, $a_{n}=0$ and the velocity and piezometric head are constant at all points. It follows that every term in an equation of the Bernoulli form

$$
\frac{v_{1}^{2}}{2 g}+h_{1}=\frac{v_{2}^{2}}{2 g}+h_{2}
$$

applies to any and all streamlines between sections 1 and 2 in plate 1(i).

For the circumstances illustrated by plate 1 (i), it has been determined that the velocity at all points in section 1 is equal to $v_{1}$. This velocity, therefore, can be represented by the symbol $V_{1}$, for $V$ has been defined as the average velocity in a total cross section. Similarly, here, $v_{2}=\mathrm{V}_{2}$. When these substitutions are made in equation 25 , the resulting equation,

$$
\frac{V_{1}^{2}}{2 g}+h_{1}=\frac{V_{2}^{2}}{2 g}+h_{2}
$$

can be described as an equation of motion for a typical streamline or an equation of motion for a streamtube which envelops the total flow.

Plate 1 (j) shows an orifice flow pattern which differs in some very important respects from the flow pattern shown in plate 1 (i). In plate $1(j)$ a diaphragm orifice is located in a pipe. Section 2 is again located in the parallel jet. Thus, as in the previous example, the velocity and the piezometric head at all points in section 2 are essentially constant. Section 1 is normal to the axis of the pipe and far enough upstream from the orifice to be removed from the zone of streamline curvature. Thus, as the streamlines are all straight 
and perpendicular to section 1 , the normal acceleration is everywhere zero, and the piezometric head is constant. However, the influence of the shear forces on the velocity distribution in section 1 can no longer be ignored. As the velocity of a viscous fluid relative to its fixed boundaries becomes appreciable, shear forces cause the fluid near the boundary to be retarded. As illustrated in plate $1(j)$, therefore, the velocities in section 1 vary with distance from the boundary.

Another effect due to the influence of shear is shown in plate $1(\mathrm{j})$. As there can be no slippage at the boundaries, the velocity of the contact fluid is always zero. On the other hand, flow approaching the upstream corners must decelerate. The apparent conflict between the condition of zero velocity at the boundary and the tendency for deceleration along the boundary is resolved by separation and the formation of an eddy zone in the corner region. The result is a modification of the flow pattern and a depreciation of flow energy. These matters obviously cannot be considered in detail at this time because the simplified equations of motion were derived on the condition that the influence of shear forces was negligible.

For many practical purposes, it is permissible to ignore certain effects of viscous and turbulent shear. For a pattern such as that in plate $1(\mathrm{j})$, for example, it is generally sufficient to ignore the nonuniformity of tangential velocities across the normal sections and to acknowledge that equation 26 is a good approximation. Under such circumstances, because it ignores the influence of transverse velocity variations, equation 26 is called a one-dimensional equation of motion.

\section{RQUATION OF CONTINUITY}

Plate $1(\mathrm{k})$ shows a typical streamtube in a steady fluid motion. The average tangential velocity at any section is $v_{s}$, and the area of a section normal to the axis of the tube at any station is $\Delta A_{n}$. The product $v_{s} \Delta A_{n}$ has already been defined as discharge, or volume rate of flow, a quantity which has no counterpart in rigid-body mechanics. The dimensions of discharge indicate that it is a measure of the time rate of passage of fluid volume through the streamtube. The product of discharge and density, therefore, is a measure of the rate of passage of fluid mass. By definition, the flow normal to the streamtube surfaces is everywhere zero. Thus, because fluid mass can be neither created nor destroyed, in a steady fluid motion the mass rate of flow $\left(\rho v_{s} \Delta A_{n}\right)$ is constant at successive sections, or, $\left(\rho v_{s} \Delta A_{n}\right)_{1}=\left(\rho v_{s} \Delta A_{n}\right)_{2}$. For a liquid flow, furthermore, the density is essentially constant. Thus, the volume rate of flow $(\Delta Q)$ at successive sections is also constant, or

$$
\Delta Q=\left(v_{s} \quad \Delta A_{n}\right)_{1}=\left(v_{s} \Delta A_{n}\right)_{2} .
$$


The discharge $(Q)$ through a total cross section between fixed boundaries is the summation of the discharges through the component subsections, or

$$
Q=\Sigma(\Delta \supsetneq)=\Sigma\left(v_{s} \Delta A_{n}\right)=\int\left(v_{s} d A_{n}\right)=V A,
$$

in which $V$ is the average velocity and $A$ is the total area of the section. Thus, because the fixed boundaries comprise limiting stream surfaces, from equations 27 and 28 , the total discharge is constant at successive sections, and

$$
Q=V_{1} A_{1}=V_{2} A_{2} \text {. }
$$

Equation 29 is the one-dimensional equation of continuity.

\section{DISCFARGE FQUATION}

Equation 29 can be applied, for example, to sections 1 and 2 in plate 1 (j). It can also be solved simultaneously with equation 26 , which is an approximate equation of motion for the same flow pattern. The result,

$$
Q=A_{1} \sqrt{\frac{2 g\left(h_{2}-h_{1}\right)}{1-\left(\frac{A_{1}}{A_{2}}\right)^{2}}} \text { or } Q=A_{2} \sqrt{\frac{2 g\left(h_{1}-h_{2}\right)}{1-\left(\frac{A_{2}}{A_{1}}\right)^{2}}}
$$

is a discharge equation in terms of area and piezometric head. As is true of equation 26, equation 30 is applicable only when the influence of the shear forces is small.

When the discharge equation is applied to a flow pattern such as that shown in plate $1(\mathrm{j})$, the area at section 2 is most conveniently expressed in terms of the area $(A)$ of the orifice and the coefficient of contraction $\left(C_{c}\right)$. By definition, and as illustrated in plate 1 (l),

$$
A_{2}=C_{c} A \text {. }
$$

Thus, from equations 30 and 31 , a common form of the discharge equation for a convergent boundary is

$$
Q=\frac{C_{c} A}{\sqrt{1-\left(\frac{C_{c} A}{A_{1}}\right)^{2}}} \sqrt{2 g\left(h_{1}-h_{2}\right)}
$$

or

$$
Q=C_{d} A \sqrt{2 g\left(h_{1}-h_{2}\right)},
$$


in which $C_{d}$ is the coefficient of discharge. From equations 32 and 33,

$$
C_{d}=\frac{C_{c}}{\sqrt{1-\left(\frac{C_{c} A}{A_{1}}\right)^{2}}}=\frac{\frac{Q}{A}}{\sqrt{2 g\left(h_{1}-h_{2}\right)}} .
$$

\section{FLOW NET}

The flow net is a pattern of streamlines and "potential" lines which indicates by its configuration the velocity distribution in a fluid flow. Flow nets can be constructed only in accordance with the following restrictions: The flow to be represented is a steady flow; the flow pattern is independent of the influence of fluid viscosity, surface tension, and elasticity; the flow pattern is independent of the influence of fluid weight (this does not preclude flow nets for completely enclosed flows in vertical planes nor certain kinds of free-surface flows). Within thelimits of the restrictions above, flow nets can be constructed for flows involving free-stream surfaces as well as those which are effectively guided by rigid boundaries. A flow net is unique for the given boundary conditions, regardless of scale (size) or any assumptions regarding fluid density, velocity, elevation, or pressure.

From hydrodynamics it is known that the equations of the lines which comprise the flow net can be determined for many two-dimensional flows and certain axisymmetric three-dimensional flows. From hydrodynamics it is also known that the requirement of negligible fluid viscosity is corequisite to the mathematical restriction of irrotationality.

Truly irrotational flow, of course, is impossible; however, as already observed, there are some kinds of fluid motion in which the influence of the complicating fluid properties is negligible. For such flows, usually involving rapidly accelerating motion and a completely enclosed or submerged flow pattern, the flow net affords a powerful means of analysis.

Whereas certain flow nets can be plotted as families of curves whose equations are developed by mathematical techniques, many others can be determined only by graphic or arithmetic successive-approximation procedures. Electric analogies, laminar-flow analogies, and other laboratory techniques facilitate the approximation procedures. Flow nets involving free-stream surfaces are more difficult to obtain than nets for totally enclosed flows. However, measurement of the free-stream profile under carefully controlled laboratory conditions is an expedient.

It is apparent that, in general, flow nets are not easily obtained. It will be made equally apparent, however, that a flow net once drawn 
for a particular boundary shape affords a universal solution for many fundamental flow characteristics. Some typical two-dimensional flow nets are shown on plate $2(a-h)$.

One of the definitive characteristics of a two-dimensional flow net is that the streamlines are so spaced that the discharge between all adjacent streamlines in the net is a constant. Furthermore, from the equation of continuity, the discharge past successive sections in each of the two-dimensional streamtubes is also constant. In plate 2 (d), $v$ is the average velocity and $\Delta n$ is the corresponding normal distance between any pair of streamlines in a typical flow net. Because $\Delta q=v \Delta n$, and because $\Delta q_{o}=\Delta q_{1}=\Delta q_{2}=\Delta q_{3}$ in plate 2 (d), it follows that $v_{0} \Delta n_{0}=v_{1} \Delta n_{1}=v_{2} \Delta n_{2}$, etc., throughout the flow net. Thus, the relative velocity at any point in the flow pattern is indicated by the distance between streamlines at that point. For example, if $v_{0}$ and $\Delta n_{0}$ are described as reference characteristics in a particular flow net, the velocity at any other point is $v=\Delta q / \Delta n=\left(v_{0} \Delta n_{0}\right) / \Delta n$, or

$$
\frac{v}{v_{o}}=\frac{\Delta n_{o}}{\Delta n}, \frac{v_{1}}{v_{0}}=\frac{\Delta n_{o}}{\Delta n_{1}}, \frac{v_{2}}{v_{o}}=\frac{\Delta n_{o}}{\Delta n_{2}}, \text { etc. }
$$

In regions of flow where acceleration is appreciable, finite values of the normal distance between streamlines provide only an approximate measure of the mean velocity between the streamlines. Further subdivision of the net, however, affords a means of improving the accuracy of the measure. The limit of the subdivision procedure occurs when $\Delta n=d n$. Then $\Delta q=d q=v d n$ is the constant infinitesimal discharge in each of an infinite number of streamtubes which make up the flow net. Actually, the decision regarding the number of streamlines to be drawn-that is, the practical limit of subdivision-is always based on a consideration of the labor involved, the accuracy attainable, and the accuracy required.

The potential lines on a flow net are a family of lines which are orthogonal (perpendicular) to the streamlines, including the effective boundaries. The potential lines are customarily drawn so that they are everywhere equal in spacing $(\Delta s)$ to the spacing of the adjacent streamlines $(\Delta n)$. Together, the conditions of perpendicularity and equivalent spacing of streamlines and potential lines govern the graphical, successive-approximations method of constructing a flow net.

In regions where the flow is uniform the meshes formed by pairs of streamlines and potential lines are squares. In regions of nonuniform flow, the meshes are imperfect squares, although individually their medians are equal and their corners form $90^{\circ}$ angles. If the net is further subdivided, however, all of the component meshes approach perfect squares as $\Delta n$ and $\Delta s$ approach $d n$ and $d s$, respectively, just as 
all curved lines approach straight lines as the length of line considered approaches zero.

Because in any local zone $\Delta s$ is equal to $\Delta n$, it follows that $\Delta s$ is a measure of the mean velocity between adjacent potential lines for the same reason that $\Delta n$ is a measure of the mean velocity between adjacent streamlines. Thus, in a uniform-flow zone, $\Delta n=\Delta s$, and

$$
\frac{v}{v_{o}}=\frac{\Delta n_{o}}{\Delta n}=\frac{\Delta s_{o}}{\Delta s}, \text { etc. }
$$

In nonuniform zones, either $\Delta n$ or $\Delta s$ might be preferable as an approximate measure of the mean velocity, depending on the characteristics of the flow pattern and the purpose of the analysis. For example, along any streamline, including the boundary, the maximum and minimum velocities are best indicated by the potential-line spacing.

Typical velocity-distribution curves, determined from the flow net for a sluice gate, are shown on plate $2(\mathrm{~g})$. Here, velocities along the floor of the channel and along the face of the sluice gate are represented as a multiple of the downstream uniform velocity $\left(V_{2}\right)$. Thus, the velocity-distribution curves shown on plate $2(\mathrm{~g})$ are plots of the relationship

$$
v=\left(\frac{\Delta s_{2}}{\Delta s}\right) V_{2},
$$

in which $v$ is the velocity at any point, $\Delta s$, is the distance between potential lines at that point, and $\Delta s_{2}$ is the distance between potential lines in the downstream, uniform-flow zone.

It has been established that the velocity variation throughout an irrotational-flow pattern is related to the piezometric-head variation. It follows that the flow net provides a means of analyzing the piezometric-head distribution throughout any flow pattern for which a net can be drawn. From the Bernoulli equation (14), when it is applied between a reference point and a general point in any irrotational-flow pattern,

$$
h-h_{0}=\frac{v_{0}^{2}}{2 g}-\frac{v^{2}}{2 g},
$$

in which $h=(p / \gamma)+z$ is the piezometric head. By dividing both sides of this equation by the velocity head at the reference point and observing that

$$
\frac{v}{v_{0}}=\frac{\Delta n_{0}}{\Delta n}=\frac{\Delta s_{0}}{\Delta s}
$$

then

$$
\frac{h-h_{o}}{\frac{v_{o}^{2}}{2 g}}=1-\left(\frac{v}{v_{o}}\right)^{2}=1-\left(\frac{\Delta n_{o}}{\Delta n}\right)^{2}=1-\left(\frac{\Delta s_{o}}{\Delta s}\right)^{2}
$$


The left-hand member of equation 37 , called the piezometric-head ratio, is dimensionless. It contains only one dependent variable $(h)$, in addition to the reference values $\left(h_{o}\right.$ and $\left.v_{o}\right)$. The terms on the righthand side of the equation indicate that the piezometric-head ratio is a function of the flow-net configuration alone. It has been established from hydrodynamics that, if one can be drawn, a flow net is unique for any given boundary conditions; in other words, there is only one flow net for a given boundary form. It follows that the piezometric-head ratio is determined for all points in a given flow pattern by the shape of its boundaries. The full significance of this conclusion is best illustrated by the observation that both the velocity ratio (eq 36) and the piezometric-head ratio are independent of the size of the boundary as well as of the fluid density, discharge, elevation, and pressure.

Typical piezometric-head distribution curves, corresponding to the velocity curves in plate $2(\mathrm{~g})$, are shown for the sluice gate in plate $2(\mathrm{~h})$. From equation 37, when it is applied between a general point and a point in the uniform flow downstream from the gate,

$$
h=\left[1-\left(\frac{\Delta s_{2}}{\Delta s}\right)^{2}\right] \frac{V_{2}^{2}}{2 g}+h_{2}
$$

in which $\Delta s_{2}, V_{2}^{2} / 2 g$, and $h_{2}$ are constants which, for any scale of drawing, fix the scale of the piezometric-head graph. Thus, the only variable in this equation is the distance $\Delta s$, which is the scaled distance between potential lines at the point where the piezometric head is $h$. For convenience, in plate $2(h)$ the piezometric head is represented as a multiple of the gate opening (b); that is, $h=C b$, where $C$ is a function of a single variable $(\Delta s)$.

Because the elevation of any point on the flow pattern shown in plate $2(\mathrm{~h})$ is readily scaled off the drawing, it follows that the pressure head at any point along the gate face or the floor is represented as the difference between the plotted piezometric head and the elevation. In this manper the flow net can be used to evaluate the total fluid-pressure force acting on any portion of the boundary.

It is emphasized that the sluice gate shown in plate $2(\mathrm{f}-\mathrm{h})$ represents a fluid motion in which the influence of viscosity is almost negligible. Also, it is observed that, in this example, the influence of fluid weight is confined to its accounting for a variation of pressure with elevation. In other words, this is the kind of flow pattern for which a flow net yields useful, practical information. Many such examples could be given. ${ }^{2}$

2 Rouse, Hunter (ed.) 1950, Engineering hydraulics: New York, John Wiley \& Sons, Ine. See p. 22, 33, 47-52, 534-542. 
The coefficient of discharge for convergent-boundary flow patterns has been defined by the relationships

$$
C_{d}=\frac{C_{c}}{\sqrt{1-\left(\frac{C_{c} A}{A_{1}}\right)^{2}}}=\frac{\frac{Q}{A}}{\sqrt{2 g\left(h_{1}-h_{2}\right)}} .
$$

The prerequisites for the applicability of this equation are essentially the same as those which determine whether the flow pattern can be represented by a flow net. In fact, a property of all flow nets-that the streamline configuration is uniquely determined by the form of the fixed boundaries-leads to the conclusion that the second member of equation 34 involves only dimensionless characteristics of the flow boundary. In other words, $C_{c}$ and, therefore, $C_{d}$, are functions of the boundary geometry alone. This conclusion is substantiated by the observation that the right-hand member in equation 34 is a particular form of the piezometric-head ratio; that is,

$$
C_{d}=\frac{\frac{Q}{A}}{\sqrt{2 g\left(h_{1}-h_{2}\right)}}=\frac{1}{\sqrt{\frac{h_{1}-h_{2}}{\frac{V^{2}}{2 g}}}},
$$

where $V$ is the average velocity through the gross orifice area and $h_{1}-h_{2}$ is the difference in piezometric heads between the two uniformflow regions designated sections 1 and 2 . Thus, $C_{a}$ has all the characteristics of any piezometric-head ratio, including those of being dependent on the form of the fixed boundaries and independent of boundary scale, fluid density, discharge, elevation, and pressure.

\section{THE EXPERIMENTAL METHOD}

A simple kind of motion which involved mainly the forces due to pressure gradient and fluid weight was examined in the preceding section. The discharge of an incompressible fluid through a plate orifice between adjacent large reservoirs was described as a practical example of this kind of motion. In general, of course, additional forces must be considered. Thus, when a fluid flows between fixed boundaries, viscous and turbulent shear stresses are involved. On the other hand, when accelerated motion with a free surface occurs, certain independent effects due to fluid weight must be considered.

Unfortunately, flow patterns which depend on the combined effects of several fluid-property forces are seldom subject to complete mathematical description. Thus, a full complement of expanded equations of motion, counterparts of the equations for a simple fluid motion 
derived in the preceding section, is not forthcoming. In this section a method of analysis which provides an indirect evaluation of the relative influence of the fluid-property forces will be considered.

\section{INFLUENCE OF SEEAR}

The effects of viscous and turbulent shear are prominent in most fluid-flow phenomena. They are manifested, usually, by their influence on the energy of flow and tis external aspects of the flow pattern. Thus, as a consequence of viscous shear, heat is generated within a moving fluid and the energy of the flow system is thereby degraded. On the other hand, shear stresses are primarily responsible for the occurrence of boundary drag forces, separation zones, eddies, and various phenomena associated with the boundary layer.

In the absence of comprehensive equations of motion, practical solutions for many problems in engineering hydraulics involve empirical adjustments of the simple equations of motion derived in the preceding section. Thus, an understanding of the complex part played by the shear forces depends, in general, on an understanding of the techniques and limitations of dimensional analysis and experiment.

\section{EXPERIMENTS AND THE THEORY OF SIMIITTUDE}

The principal purpose of experiment is to determine the relative influence of each of the variables which govern the occurrence of a phenomenon. For occurrences lacking a general analytical solution, however, the identification of the independent variables is often as obscure as the evaluation of their influence. Frequently, therefore, an auxiliary purpose of experimental observations is to isolate the effective variables. Many practical problems have been satisfactorily solved by semi-empirical methods based on experimental data. However, it is emphasized that empirical solutions lack generality by their failure to recognize the exact functional relationship between the primary variables.

The variables involved in fluid motion are contained in one of following categories: boundary conditions; kinematic or flow characteristics, including acceleration and velocity (or discharge); and dynamic characteristics, or the forces which cause motion. One of the most powerful tools of modern fluid mechanics is that which utilizes dimensionless ratios to describe boundary geometry, flow characteristics, and force characteristics. The dimensionless-ratio technique provides a means of correlating similar phenomena without restriction as to scale, flow, or fluid properties. It is fundamental to the planning and execution of experimental programs, to the analysis of experimental data, and to the application of solutions which are based on experimentation. 
The general Newtonian equation of motion for fluids has already been expressed in the symbolic form (eq 3), $F_{p} \rightarrow F_{g} \rightarrow F_{r} \rightarrow F_{\sigma} \rightarrow F_{\varepsilon}=$ Ma.

Plate 2 (i-l) illustrates the significance of such an equation when applied to the motion of a small element of fluid wherein the effects of $F_{\sigma}$ and $F_{\varepsilon}$ are negligible. Thus, in plate $2(\mathrm{j})$,

$$
F_{p} \nrightarrow F_{g} \nrightarrow F_{\tau}=M a \text {. }
$$

The size of the polygon which represents this force system depends on the scale selected for the drawing and the relative magnitude of the forces involved. It follows that the force picture at a corresponding point in a completely similar flow pattern might be represented to the same scale by plate $2(\mathrm{k})$. However, if every term in equation 39 were divided by the resultant inertial reaction $(M a)$,

$$
\frac{F_{p}}{M a}+\frac{F_{g}}{M a}+\frac{F_{\tau}}{M a}=1
$$

the force systems shown by the similar polygons in plate $2(\mathrm{j}, \mathrm{k})$ might both be represented by the unit polygon shown in plate 2 (l). At corresponding points in all truly similar flow patterns such dimensionless force polygons are identical.

The magnitude of each of the ratios in equation 40 is a relative measure of the part played by a particular accelerative force. Each of the ratios, therefore, has obvious significance as a criterion of similarity. For example, the first ratio is a measure of the influence of the pressure force relative to the total inertial reaction. It is evident that when this and each of the other ratios are fixed, the force pattern and, therefore, the motion pattern, are determined. It follows that two or more flow patterns are completely similar when their boundaries are geometrically similar and the corresponding force ratios are identical. Thus, dynamic similarity is independent of the, size of the boundaries, the magnitudes of the velocities, or the physical properties of the fluids involved.

Equation 40 and plate 2 (1) have been used to demonstrate the significance of dimensionless force ratios as a means of describing a particular fluid motion. Equivalent ratios, which are proportional to the reciprocals of the square roots of the ratios in equation 40 , are widely accepted as general criteria of similarity. The first of these is the Euler.number (E),

$$
\mathbf{E}=\frac{V}{\sqrt{\frac{2 \Delta p}{\rho}}}
$$


in which $V$ is a typical average velocity and $\Delta p$ is a significant pressure difference. The Euler number is derived from the pressureforce ratio, the first term in equation 40 . Thus, the Euler number is a measure of the relative influence of the pressure force.

Similarly, a ratio which is a measure of the influence of the gravity force and which is derived from the second term in equation 40 is called the Froude number (F). For free-surfaee flows, the accepted form of this ratio is

$$
\mathbf{F}=\frac{V}{\sqrt{g L}}
$$

in which $L$ is a significant length.

In the third term of equation 40 ,

$$
\frac{F_{r}}{M a}=\frac{F_{\mu}}{M a}+\frac{F_{\eta}}{M a}=\frac{F_{\mu}}{M a}+\text { Constant }
$$

in which $F_{\mathbb{a}} / M a$ is a constant because turbulent shear forces are proportional to the total inertial reaction. It follows that

$$
\frac{F_{p}}{M a}+\frac{F_{s}}{M a}+\frac{F_{\mu}}{M a}=\text { Constant. }
$$

The third term in equation 43 represents the relative influence of the independent viscous shear force. The accepted form of the ratio which is derived from this term is called the Reynolds number $(\mathbf{R})$,

$$
\mathbf{R}=\frac{V L \rho}{\mu} \text {. }
$$

Similar ratios which have been formulated to describe the surfacetension and elastic forces are called the Weber and Cauchy (or Mach) numbers, respectively.

Inasmuch as the Euler, Froude, and Reynolds numbers are proportional to the dimensionless ratios in equation 43 , it follows that complete similarity of two or more flow patterns will prevail when their boundaries are geometrically similar and their Euler, Froude, and Reynolds numbers are equal.

Of the ratios in equation 43 , the Euler number (E) has special significance as a general flow parameter. Because it involves no fluid property other than density, $\mathbf{E}$ is the sole criterion of similarity for motion which, from hydrodynamics, is described as ideal. Thus, for an enclosed flow in a horizontal plane with negligible influence due to shear, inertial reactions are a function of pressure forces alone. For any given boundary conditions, therefore, $\mathbf{E}$, representing a ratio of 
a typical inertial reaction to a typical pressure force, is a constant. In other words, for ideal-fluid motion, $\mathbf{E}$ is a function of boundary conditions alone. On the other hand, if forces other than pressure are partly responsible for the total inertial reaction, the magnitude of $\mathbf{E}$ reflects the relative influence of the other forces. As all the other ratios are related to the fluid properties, the Euler number is appropriately selected as a dependent ratio. This leads to the statement of a general principle of similitude: Values of $\mathbf{E}$ for two or more flow patterns are identical, and the patterns, therefore, are exactly similar if their boundary conditions are similar and if the corresponding values of the fluid-property parameters, such as $\mathbf{F}$ and $\mathbf{R}$, are equal.

A significant example of the Euler number is the coefficient of discharge described on pages 24 and 28. Other examples are the uniform-flow resistance coefficients, various minor-loss coefficients, drag-force coefficients, etc. From equation 38,

$$
C_{d}=\frac{\frac{Q}{A}}{\sqrt{2 g\left(h_{1}-h_{2}\right)}}=\frac{V}{\sqrt{\frac{2}{\rho}\left(p_{1}-p_{2}\right)}}=\mathbf{E} .
$$

To all those conclusions concerning $C_{d}$ which were derived previously from the piezometric-head ratio analogy and the flow-net characteristics, there are now added the limitations imposed by the influence of the fluid-property forces. Thus $C_{d}$, obviously an important flow parameter, is now defined as a function of not only the boundary geometry but also the several fluid-property parameters derived in this section. ${ }^{3}$

\section{INFLUENCE OF A FREE SURFACE}

Certain kinds of free-surface flow patterns, such as the sluice gate (pl. $2, \mathrm{f}-\mathrm{h}$ ) and the simple measuring weir, are free of independent weight-force effects. In other words, for those flow patterns which are uniquely determined by the fixed boundaries, the only influence of fluid weight is to account for a variation of pressure with elevation.

Under other conditions, as illustrated by the brink at the end of a sloping channel or the orifice at the end of a pipe, the weight force can have an independent influence on the flow pattern. For example, when a fluid discharges from an'orifice into a space occupied by another fluid of different density, the trajectory of the jet and many related characteristics of the flow pattern depend on the velocity of approach, the specific weight of the fluid flowing, and the specific weight of the surrounding fluid, as well as the boundary geometry.

\footnotetext{
Rouse, Hunter, 1946, Elementary mechanies of flutds: New York, John Wley \& Sons, Inc. The reader is urged to study the sequence of $C_{d}$ curves represented by figures $31,52,85$, and 129 .
} 
The effect of the weight force in this example is to deflect the jet upward or downward, depending on its effective weight or buoyancy relative to the.surrounding fluid. The relative magnitude of this effect depends on the ratio of the inertia of the jet to the weight force. Such a ratio, of course, is the Froude number. However, for an application of this kind, $\mathbf{F}$ must be written in the more general form,

$$
\mathrm{F}=\frac{V}{\sqrt{\frac{\Delta \gamma}{\rho} L}},
$$

in which $\Delta \gamma$ is the difference in specific weights of the two fluids involved and $\rho$ is the density of the fluid flowing.

Practical problems related to free-surface flows are seldom subject to complete analytical solution. By means of the Froude criterion, however, along with the other dimensionless force ratios described previously, satisfactory solutions based on experiment can be achieved.

\section{RECAPITULATION}

A preceding section was principally concerned with a simple kind of fluid motion in which the only effective forces were those due to. pressure difference and weight. The main objective of that section was to derive equations which constitute a mathematical description of the motion. The most important equations derived were the Bernoulli equation, which defines the relationship between velocities, pressures, and elevations throughout the flow pattern; the onedimensional equation of motion, which describes the variation in the average flow characteristics between sections of essentially uniform motion; the one-dimensional equation of continuity; and the discharge equation. In addition, the flow net was introduced as a valuable analytical tool and a means of demonstrating the significance of the equations of motion.

It is now acknowledged that neither the simple equations of motion nor the flow net are theoretically compatible with the occurrence of shear forces. It is also acknowledged that, in general, it is not possible to derive expanded equations of motion which adequately define the influence of shear and certain free-surface flow characteristics due to fluid weight. Thus, for many problems involving appreciable effects due to viscosity, weight, or any other fluid property, solutions based on field or laboratory experiments must be sought. For this purpose the dimensionless-ratio technique of specifying boundary characteristics (a length ratio, usually), flow characteristics (a coefficient of discharge, piezometric-head ratio, efficiency, energy-loss coefficient, or some other form of the Euler number), and fluid-property. 
force characteristics (the Froude, Reynolds, Weber, and Cauchy numbers) has been described. Flow-pattern descriptions based on dimensionless ratios are completely independent of restrictions regarding scale, discharge, pressure, or fluid properties. 'Thus, dimensionless ratios are the basis for the general principle of similitude which governs all experimental investigations.

\section{EXAMPLE}

The manner in which the principle of similitude can be used to guide the planning and execution of an experimental program is demonstrated by the following example. It is proposed to make laboratory tests on a diaphragm orifice. The purpose of the tests is to determine the discharge characteristics of an 8-inch thick-plate metering orifice in a 16-inch pipeline carrying crude oil. The laboratory tests are to be made in a 4-inch pipe carrying water. The principal objective of the investigation is to define the coefficient of discharge $\left(C_{d}\right)$ for a full range of oil-pipeline (prototype) flows. This objective is incidental, of course, to the requirement that the flow pattern in the pipeline be exactly simulated by the flow pattern created in the experimental setup.

The scale ratio of lengths in this example is determined by the ratio of pipe diameters, 1:4. In order to achieve geometric similarity, therefore, the laboratory orifice must be one-fourth as large as the pipeline orifice, or 2 inches in diameter. Similarly, all other length characteristics, such as plate thickness, rounding radius, and piezometer locations, must be reproduced in accordance with the 1:4 length-scale ratio.

In order to obtain completely similar flow patterns in the vicinity of the geometrically similar orifices, the relative influence of each of the effective independent forces must be the same. In this example, viscous shear forces are involved in the occurrence of the boundary layer upstream from the orifice and in all the consequent effects described on page 22. Because the flow pattern is entirely enclosed, however, the weight force does not have an independent influence. Surface-tensian forces are absent because there are no fluid interfaces, and elastic forces are negligible because both of the fluids involved are liquids.

It follows that, for similar boundary conditions, pressure differences are functions of viscous shear forces and accelerations. Therefore, ratios of the Euler variety, including the coefficient of discharge, are functions of the Reynolds number alone. In other words, complete similarity of the flow pattern (including turbulence) will be achieved and the discharge coefficients for the two orifices will be equal when their Reynolds numbers are equal. It is this condition which de- 
termines the range of laboratory test conditions required to define $C_{d}$ for a full range of pipeline discharges.

Thus, for similar flow patterns and equal coefficients of discharge,

$$
\begin{aligned}
\mathbf{R}_{w \text { (water) }} & =\mathbf{R}_{o \text { (oil), or, }} \\
\left(\frac{V L_{\rho}}{\mu}\right)_{w} & =\left(\frac{V L_{\rho}}{\mu}\right)_{o},
\end{aligned}
$$

in which $V$ is usually defined as the average velocity based on the gross area of the orifice, and $L$ is the diameter of the orifice. From this relationship, the ratio of velocities for similar flow conditions is

$$
\frac{V_{w}}{V_{o}}=\left(\frac{L_{o}}{L_{w}}\right)\left(\frac{\rho_{o}}{\rho_{w}}\right)\left(\frac{\mu_{w}}{\mu_{o}}\right)
$$

and, because discharge is proportional to the product of a velocity and an area (or the square of a length),

$$
\frac{Q_{w}}{Q_{o}}=\frac{A_{w} V_{w}}{A_{o} V_{o}}=\left(\frac{L_{w}}{L_{o}}\right)^{2}\left(\frac{L_{o}}{L_{w}}\right)\left(\frac{\rho_{o}}{\rho_{w}}\right)\left(\frac{\mu_{w}}{\mu_{o}}\right) .
$$

Therefore, the discharge of water in the laboratory setup required to simulate a given rate of flow in the pipeline can be expressed in terms of the length ratio, the density ratio, and the viscosity ratio, or,

$$
Q_{w}=\left[\left(\frac{L_{w}}{L_{o}}\right)\left(\frac{\rho_{o}}{\rho_{w}}\right)\left(\frac{\mu_{w}}{\mu_{o}}\right)\right] Q_{o} .
$$

In this example, $L_{w o} / L_{o}=1 / 4 . \quad$ Furthermore, the density and viscosity ratios are virtually fixed because, for given fluids, these properties can be altered only slightly (by changes in temperature). Thus, equation 48 is of the form $Q_{w}=K Q_{o}$, and $K$ is essentially constant.

The example would have been considerably complicated if the metering orifice had been located at the end of the pipeline. Because the orifice would then discharge freely into the atmosphere, fluid weight would be added to the independent variables already considered. Thus, the Froude number would be an additional criterion governing the performance of the laboratory investigation. Under certain conditions this added requirement makes complete similarity impossible to achieve.

The difficulty of satisfying more than one fluid-property criterion is easily demonstrated. From the requirement that the Froude numbers be equal for similar flow patterns,

$$
\left(\frac{V}{\sqrt{g L}}\right)_{\omega}=\left(\frac{V}{\sqrt{g L}}\right)_{0}
$$


or,

$$
\frac{V_{v 0}}{V_{0}}=\left(\frac{L_{v 0}}{L_{0}}\right)^{3 / 2}
$$

whence,

$$
Q_{v}=\left(\frac{L_{v}}{L_{0}}\right)^{5 / 2} Q_{0}
$$

For similarity with respect to viscous forces as well as the weight force, equation 50 must be equivalent to equation 48 . Solving the two equations simultaneously,

$$
\left(\frac{L_{w}}{L_{0}}\right)^{5 / 2}=\left(\frac{L_{w}}{L_{0}}\right)\left(\frac{\rho_{0}}{\rho_{w}}\right)\left(\frac{\mu_{w}}{\mu_{0}}\right)
$$

or,

$$
\frac{\mu_{v o} / \rho_{w}}{\mu_{0} / \rho_{0}}=\left(\frac{L_{w}}{L_{0}}\right)^{3 / 2}
$$

Equation 51 places a definite restriction on the physical properties of the fluids involved in the investigation. In fact, assuming that the length ratio and the experimental fluid are fixed within narrow limits by practical considerations, equation 51 imposes an impossible condition on the investigation. Thus, because of the small range in magnitude represented by the viscosities and densities of ordinary fluids, it is a conclusion of general significance that it is impossible to satisfy simultaneously more than one of the fluid-property criteria.

Fortunately, the apparent difficulty in attempting to satisfy all of the similitude criteria is not a hopeless barrier to experimental investigations. It is recalled that the Reynolds number, for example, is proportional to the ratio of a typical inertial reaction to a typical viscous-shear force. The relative influence of $F_{\mu}$, therefore, diminishes. as $\mathbf{R}$ increases. Experiments show that the effect of viscosity on $C_{d}$ for most sharp-edged orifices is negligible at values of $\mathbf{R}$ above a critical value of $100,000^{4}$ (not an unusually high value, as it corresponds, for example, to the discharge of water at $70^{\circ} \mathrm{F}$ at 6 feet per second through a 2 -inch orifice). Similarly, the influence of the weight force diminishes as the Froude number increases, becoming negligible at a comparatively low critical value of $\mathbf{F} .^{5}$

In the range wherein variations in the magnitude of a given fluidproperty criterion produces negligible changes in the flow pattern,

\footnotetext{
1 Rouse, Hunter, 1946, Elementary mechanics of fluids: New York, John Wiley \& Sons, Inc. See fig. 129, p. 256.

Ibid, fig. 52, p. 105.
} 
that criterion can be ignored as a condition for similitude. Thus, it is possible that neither viscous forces nor weight forces have an appreciable effect on the coefficient of discharge within the full range of the pipeline flows. If this were true, the coefficient of discharge would be a function of boundary conditions alone, and the laboratory program to determine $C_{a}$ for the metering orifice might actually consist of a single test. However, that test could not be made with assurance until a series of tests had established the lower critical values for both $\mathbf{F}$ and $\mathbf{R}$. It is apparent, therefore, that the fluid-property parameters are useful not only as similitude criteria but also as means of defining the effective range of influence of the various fluid-property forces. The practical theory of model testing makes use of the fact that a single force can have a dominant, although not an exclusive, influence on the flow pattern.

\section{THE ENERGY EQUATION}

\section{REVIEW OF THE BERNOULLI EQUATION}

One of the fundamental concepts of fluid mechanics and the basis for many of the practical formulas of engineering hydraulics is the energy equation. To bring a detailed consideration of the energy concept into this discussion requires first a review of certain steps taken in the derivation of the equations for a simple fluid motion.

Plate 1 (c) shows the small element of fluid which, on page 13, was considered to be undergoing accelerated motion because of a combination of pressure and weight forces. It is pertinent now to discuss certain characteristics of this element which make it possible to associate with it some of the familiar concepts of particle mechanics. The elementary volume of fluid shown in plate 1 (c) was ass r med to be large enough to be represented by steady-motion characteristics in a turbulent flow. On the other hand, it was assumed to be small enough that pressure forces could be accurately expressed in terms of a mean pressure intensity, pressure gradients could be assumed constant over the length of the element, and velocity characteristics could be described in terms of the velocity at the mass center of the element. Thus, as the volume of the element $(\Delta s \Delta A)$ was, allowed to approach the differential quantity $d s d A$, the motion characteristics of the element were described as the steady-motion characteristics at a point. They might also be described as the characteristics of a fluid particle.

Because fluid flow is a continuous process, it is often convenient to consider the fluid particle as the instantaneous image of the moving fluid contained in a particular space in the flow pattern. Thus, equations of motion for a fluid particle can be related to the rate of 
flow through the space designated as its location. The volume of the element, for example, is

$$
d \forall=d s d A=v d t d A=d Q d t
$$

in which $v$ is the mean velocity in the differential length $(d s)$ of the infinitesimal streamtube on which the element is located. It follows that the weight of a fluid particle $(d W)$ can be expressed as

$$
d W=\gamma d \forall=\gamma d Q d t .
$$

An equation for the simple fluid motion represented by plate $1(\mathbf{a}-\mathbf{c})$ is equation 8 ,

$$
-\frac{d p}{d s}(\Delta s \Delta A)-\gamma(\Delta s \Delta A)=\rho a_{s}(\Delta s \Delta A) .
$$

Collecting terms in the left member of this equation, substituting the equivalent expression for $a_{s}$ from equation $1,{ }^{6}$ and replacing $\Delta s \Delta A$ with the differential volume $(d s d A)$,

$$
-\left[\frac{d}{d s}(p+\gamma z)\right] d s d A=\frac{\rho}{2}\left[\frac{d v^{2}}{d s}\right] d s d A .
$$

Reviewing the steps taken in obtaining equations 8 and 54 from Newton's fundamental equation, it appears that they both represent, on the left, the resultant of the external forces acting on the fluid element in the $s$ direction, and, on the right, the total inertial reaction in the same direction.

Continuing with the particle analogy, if $d W$ is substituted for $\gamma d s d A$ in equation 54 , and terms are rearranged,

$$
\left[\frac{d}{d s}\left(\frac{v^{2}}{2 g}+\frac{p}{\gamma}+z\right)\right] d W=0 .
$$

Equation 55 states simply that the $s$ gradient (rate of change with respect to $s$ distance) of the quantity in parentheses is zero. If the gradient is zero, it follows that the differential of the quantity in parentheses is zero over a small distance $(d s)$, or,

$$
\left[d\left(\frac{v^{2}}{2 g}+\frac{p}{\gamma}+z\right)\right] d W=0 .
$$

The process of determining the differential of the quantity in parentheses consisted of multiplying the gradient by the distance $(d s)$. It

\footnotetext{
- The subseript on $v_{a}$ in the definition of $a$, will henceforth be omitted. By definition, $v_{n}$ is equal to zero, and $v_{\mathrm{a}}$ is therefore equal to the total velocity, $v$.
} 
is this operation which converts the vector force-inertia relationships in the preceding equations into a scalar work-energy relationship (eq 56). The conversion might have been more apparent had the operation been performed while the differential equation was in the form shown by equation 54. In that procedure, multiplying the left-hand term, a force, by $d s$ converts it to a measure of the work performed by the external forces during the time interval $(d t)$ represented by the average velocity $(v)$ and the distance $(d s)$. Concurrently, the right-hand term, $\left[d\left(\rho v^{2} / 2\right)\right] d s d A=d\left[d W\left(v^{2} / 2 g\right)\right]$, is a measure of the change in kinetic energy of the particle whose weight is $d W$.

Equation 56, therefore, is an expression of the familiar work-energy principle of mechanics. A convenient interpretation of the concept of work-energy equivalence and an examination of the units involved in equation 56 lead to the practice of referring to the parenthetical quantity as the total energy per pound of the fluid flowing. Thus, equation 56 is interpreted to mean that the total energy of the particle of weight $(d W)$ remains unchanged over the distance interval (ds). It follows that the quantity in parentheses is a constant during the interval, or,

$$
\left(\frac{v^{2}}{2 g}+\frac{p}{\gamma}+z\right) d W=\text { Constant. }
$$

Equation 57 is simply the integral (along the streamline) of equation 56. When it is divided by $d W$ it becomes the familiar Bernoulli equation,

$$
\left(\frac{v^{2}}{2 g}+\frac{p}{\gamma}+z\right)=H
$$

in which $H$, the constant of integration, is the total energy per pound of fluid flowing.

In review, the Bernoulli equation is a description of a simple fluid motion along a streamline. In its customary form (eq 58), it states that the sum of three quantities, collectively defined as the total energy per pound of fluid $(H)$, remains constant during the motion. Individually, the quantities which constitute $H$ may be either a measure of work performed on the fluid or a change in its mechanical energy. Thus, the Bernoulli equation states that the total energy or "work potential" of 1 pound of fluid in motion along a streamline remains constant regardless of transformations due to changes in velocity, pressure, or elevation.

It has already been established (p. 16) that the Bernoulli equation could be assumed to apply to an entire flow pattern as long as fluid viscosity is ignored. However, the practical objective is a general equation of motion which includes terms to account for the influence of viscous and turbulent shear. 


\section{GENTRAL ENFRGY FQUATION}

As observed previously the influence of viscous and turbulent shear is manifested by its effect on the energy of flow and by its effect on the external aspects of the flow pattern. Unfortunately, general equations of motion which describe these effects are not yet available. Thus, a practicable general energy equation is based on a simple modification of the procedure leading to the Bernoulli equation.

The equation of motion for a single streamline (eq 56), modified to acknowledge the loss of energy, takes the form

$$
\left[d\left(\frac{v^{2}}{2 g}+\frac{p}{\gamma}+z\right)\right] d W=d H d W
$$

in which $d H$ is the differential loss in total energy head in the distance $d s$. From equation 53 the term $d W$ in equation 59 is equivalent to $\gamma d Q d t$, in which $d Q$ is the differential flow through the elementary streamtube which contains and defines the particle represented by $d W$. Because $d Q$ is constant in a steady flow, equation 59 is readily integrated with respect to distance along the streamline. Thus, between any two points designated 1 and 2 on a streamline,

$$
\left(\frac{v_{1}{ }^{2}}{2 g}+\frac{p_{1}}{\gamma}+z_{1}\right) d W-\left(\frac{v_{2}{ }^{2}}{2 g}+\frac{p_{2}}{\gamma}+z_{2}\right) d W=\left(H_{1}-H_{2}\right) d W .
$$

In order to obtain an equation which describes the total flow between enclosing boundaries, it is necessary to integrate equation 60 over the total cross-sectional areas which contain points 1 and 2 . The difficulty of performing this operation is made apparent by recalling that $d W=\gamma d s d A=\gamma v d t d A$. It is evident that integration with respect to area must take into account the fact that the velocity, in general; is variable over the cross section. Therefore, unless the velocity is essentially constant or is analytically defined as a function of area, the indicated integration is impossible. The assumption of constant velocity across a section between solid boundaries would be inconsistent with the known effects of viscosity. On the other hand, explicit analytical relationships between velocity and area are impossible to formulate except for a few classic examples of uniform motion.

Not only does variable velocity hinder integration over total cross sections, but, as a result of boundary nonuniformities, a nonhydrostatic pressure variation may present a similar obstacle. However, if it is assumed that the integration can be performed over a section of parallel flow, which is usually possible, the pressure distribution can be assumed to be hydrostatic-that is, the sum of the quantities $p / \gamma$ and $z$ is constant-and this obstacle is removed. Thus, if $\gamma d Q d t$ is 
:substituted for $d W$ in equation 60, and if both sides of the equation are divided by $d t$, integration with respect to the discharge $(Q)$ in the total area gives

$$
\begin{aligned}
& {\left[\gamma \int^{\Theta}\left(\frac{v_{1}{ }^{2}}{2 g}\right) d Q+Q \gamma\left(\frac{p_{1}}{\gamma}+z_{1}\right)\right]-} {\left[\gamma \int^{Q}\left(\frac{v_{2}{ }^{2}}{2 g}\right) d Q+\right.} \\
&\left.Q \gamma\left(\frac{p_{2}}{\gamma}+z_{2}\right)\right]=\gamma \int^{Q}\left(H_{1}-H_{2}\right) d Q .
\end{aligned}
$$

Incidentally, the foregoing division by $d t$ converts the work-energy relationship into a power relationship. Thus, each of the expressions in brackets on the left-hand side of equation 61 is a measure of the total power of the flowing fluid. The expression on the right-hand side, therefore, is a measure of the power "lost" between the designated cross sections.

If equation 61 is divided by the quantity $Q_{\gamma}$, it again becomes an energy equation, and the units of every term in the equation are foot-pounds per pound (or feet) of the fluid flowing. Thus,

$$
\frac{1}{Q} \int^{\odot}\left(\frac{v_{1}^{2}}{2 g}\right) d Q+\left(\frac{p_{1}}{\gamma}+z_{1}\right)-\frac{1}{Q} \int^{\Theta}\left(\frac{v_{2}{ }^{2}}{2 g}\right) d Q-\left(\frac{p_{2}}{\gamma}+z_{2}\right)=\frac{1}{Q} \int^{\Theta}\left(H_{1}-H_{2}\right) d Q .
$$

Defining the right-hand member of this equation as the average energy (head) loss $\left(H_{L}\right)$ between sections 1 and 2 , and substituting the piezometric-head symbol $(h)$ for $(p / \gamma)+z$,

$$
\frac{1}{Q} \int^{Q}\left(\frac{v_{1}{ }^{2}}{2 g}\right) d Q+h_{1}-\frac{1}{Q} \int^{Q}\left(\frac{v_{2}^{2}}{2 g}\right) d Q-h_{2}=H_{L} .
$$

Equation 62 is a general energy equation which is restricted only by the assumption of hydrostatic pressure distribution at the designated .cross sections. The head-loss term $\left(H_{L}\right)$ ordinarily requires evaluation on the basis of experiment.

In many practical applications the integration involved in the velocity-head terms in equation 62 can be approximated by a numerical summation. Thus,

$$
\frac{1}{Q} \int^{Q}\left(\frac{v^{2}}{2 g}\right) d Q \approx \frac{1}{Q} \sum^{Q}\left(\frac{v^{2}}{2 g} \Delta Q\right)
$$

Fram this, the average kinetic energy or velocity head in a total cross section can be defined as the discharge-weighted mean of the velocity heads in its constituent subsections. Each subsection is taken to represent a zone of uniform velocity. This process is conveniently adapted to the practice of determining discharge by means of currentmeter or pitot-tube velocity traverses. 
The minor importance of the kinetic-energy terms in certain applications of the general energy equation does not justify involved methods of accounting for the velocity variation in a cross section. For those applications it is customary to approximate the true average kinetic energy in terms of the average velocity in the section $(V)$. The result is a modification of equation 62 which is another form of the one-dimensional equation of motion (eq 26),

$$
\left(\frac{V_{1}^{2}}{2 g}+h_{1}\right)-\left(\frac{V_{2}^{2}}{2 g}+h_{2}\right) \approx H_{L} .
$$

The error inherent in the velocity-head terms of equation 64 is often acknowledged by means of a coefficient $(\alpha)$,

$$
\left[\alpha_{1}\left(\frac{V_{1}^{2}}{2 g}\right)+h_{1}\right]-\left[\alpha_{2}\left(\frac{V_{2}^{2}}{2 g}\right)+h_{2}\right]=H_{L} \text {. }
$$

$\alpha$, of course, is not an independent expression of the influence of velocity nonuniformity. It can be evaluated only in terms of the integral or summation definitions of the true average velocity head, equations 63 and 65 . Thus,

$$
\alpha=\frac{1}{Q V^{2}} \int^{Q}\left(v^{2} d Q\right) \approx \frac{1}{Q V^{2}} \sum^{Q}\left(v^{2} \Delta Q\right) .
$$

Equation 65, better than any other, demonstrates that the main objective of this discussion has been accomplished. The result is a general energy equation involving separate terms to account for both effects of shear- $\alpha$ as a measure of its influence on the flow pattern, and $H_{L}$ as a measure of its energy-dissipating effect.

\section{THE MOMENTUM - EQUATION}

Numerous problems arise in applied fluid mechanics which are not readily or completely defined by the energy equation. The solution of many of these problems can be accomplished by means of an independent form of the equation of motion which does not require knowledge of the energy losses due to internal viscous shear. This is known as the momentum equation. As both the momentum equation and the energy equation are derived from Newton's fundamental equation of motion, it is necessary to examine the derivations carefully in order to establish their independence.

A common starting point for the derivation of both the energy equation and the momentum equation is the differential equation given on page 38 ,

$$
-\left[\frac{d}{d s}(p+\gamma z)\right] d s d A=\frac{\rho}{2}\left[\frac{d v^{2}}{d s}\right] d s d A .
$$


Equation 54 is a statement of Newton's second law as applied to the accelerated motion of a fluid element. The left-hand member, for the conditions represented by plate $1(\mathrm{a}-\mathrm{c})$, is the resultant accelerative force, and the right-hand side is the total inertial reaction in the direction of the total-velocity vector. Equation 54 is a vector equation because force and inertial reaction are vector quantities.

It has been demonstrated that the work-energy significance of the Bernoulli equation resulted from the fact that, in its derivation, both sides of equation 54 were multiplied by the distance $(d s)$. This operation converted force to work and inertial reaction to change in kinetic energy. Both work and energy are scalar quantities. Thus, the Bernoulli equation resulted from the integration of a work-energy equation over a streamline distance without regard for the change in direction of the $s$ distance.

It is pertinent to recall that the effects of the shear forces were ignored in the derivation of equation 54. Subsequently, however, in the derivation of the general energy equation, a term which would compensate for the work done by the internal viscous shear was included.

In the derivation of the momentum equation, it is convenient to replace the left-hand member of equation 54 with one symbol which represents the resultant of all external forces. Thus, in the form

$$
d F_{s}=\frac{\rho}{2}\left[\frac{d v^{2}}{d s}\right] d s d A
$$

the quantity $d F_{s}$ is assumed to include the boundary shear force as well as the boundary pressure force and the weight force. It does not include internal shear forces, because these are mutually canceling. Apparently, therefore, the independence of the energy and momentum equations is related to the fact that the first involves the internal viscous shear forces and the second involves the external boundary shear force. ${ }^{7}$

From the calculus, $d v^{2} / d s=2 v(d v / d s)$, where $d v / d s$ is the gradient of the total velocity in the $s$ direction. When this is substituted in equation 67 ,

$$
\frac{\rho}{2}\left[\frac{d v^{2}}{d s}\right] d s d A=\rho v\left(\frac{d v}{d s}\right) d s d A,
$$

or,

$$
d F_{\mathrm{s}}=\rho v d v d A=\rho d Q d v,
$$

in which $d Q$ is the rate of flow in the infinitesimal streamtube which contains the fluid element and $d v$ is the change in velocity over the

The boundary shear force is not considered in the derivation of the work-energy equations because the relative velocity of the fluid at the boundary is zero, and therefore the work done by the shear force is also zero. 
differential distance $(d s)$ which is the length of the element. Equation 68 is still a force-inertia equation. Therefore, it is still a vector equation.

Because motion along a streamline can involve a change in the direction as well as the magnitude of the force and velocity terms in equation 68, an adequate algebraic description of accelerated motion involves similar equations in each of the fixed coordinate directions. Thus,

and

$$
\begin{aligned}
& d F_{x}=\rho d Q d v_{x}, \\
& d F_{y}=\rho d Q d v_{y} \\
& d F_{z}=\rho d Q d v_{z} .
\end{aligned}
$$

The quantity $\rho d Q d v$ in equations 68 and 69 has been described as the inertial reaction of an elementary fluid particle. The product $\rho d Q$ is equivalent to $d M / d t$, where $d M$ is the mass of the particle. If this substitution is made in the $x$ form of equation 69 , for example,

$$
\rho d Q d v_{x}=\frac{d}{d t}\left(M v_{x}\right)
$$

the right-hand member acquires a new significance. In this form it is commonly described as the rate of change of $x$ momentum or the change of $x$-momentum flux in the flow distance $d s$. This is the origin of the name "momentum equation."

For practical applications the momentum equation must be extended to a finite reach and a finite cross section. The operations required for this extension involve double integration of the differential equation of motion-first with respect to distance along the streamline and then with respect to the area of the cross section. Similar operations were involved in the derivation of the general energy equation. This time, however, a vector relationship is involved, and the integration procedures must be performed for each of the significant coordinate directions. In the following steps the $x$ direction has been selected as typical.

Because $d Q$ in equation 69 is constant, integration with respect to distance is readily accomplished. Thus, if the $x$-distance integral of $d F_{x}$ is represented by $\Delta F_{x}$,

$$
\Delta F_{x}=\rho d Q v_{x_{2}}-\rho d Q v_{x_{1}} .
$$

Because all internal forces are canceling, $\Delta F_{x}$ is simply the resultant of the external forces on the finite reach of streamtube between sections 1 and 2. 
The next step is to integrate equation 71 over the total areas at sections 1 and 2. Substituting $d Q=v d A$,

$$
\int \Delta F_{x}=\rho \int^{A_{2}} v_{x_{2}} v_{2} d A_{2}-\rho \int^{A_{1}} v_{x_{1}} v_{1} d A_{1},
$$

it is apparent that integration depends again (see page 40) on an integrable relationship between velocity and area. Thus, a general solution of equation 82 is prevented by the fact that explicit analytical relationships between $v$ and $A$ are seldom attainable. The left-hand member, however, can be simplified to $\Sigma F_{x}$, a symbol which represents the resultant of the external forces acting in the $x$ direction on the total volume of fluid between sections 1 and 2 . Thus, general momentum equations in the fixed coordinate directions are

and

$$
\begin{aligned}
& \Sigma F_{x}=\rho \int^{Q} v_{x_{2}} d Q-\rho \int^{Q} v_{x_{1}} d Q, \\
& \Sigma F_{y}=\rho \int^{Q} v_{y_{2}} d Q-\rho \int^{Q} v_{y_{1}} d Q,
\end{aligned}
$$

$$
\Sigma F_{z}=\rho \int^{Q} v_{z_{2}} d Q-\rho \int^{Q} v_{2_{1}} d Q
$$

The integrations implied by equation 73 can be approximated by the numerical summation

$$
\Sigma F_{x} \approx \Sigma^{Q}\left(\rho v_{x_{2}} \Delta Q\right)-\Sigma^{Q}\left(\rho v_{x_{1}} \Delta Q\right),
$$

in which the quantity $\rho v_{x} \Delta Q$ represents the momentum flux through a constituent subsection assumed to be located in a zone of essentially uniform velocity.

Further paralleling the various forms of the general energy equation, a typical one-dimensional form of the momentum equation is

$$
\Sigma F_{x}=Q_{\rho} \beta_{2} V_{x_{2}}-Q_{\rho} \beta_{1} V_{x_{1}}
$$

in which $V$ is the average velocity and $\beta$ is a coefficient which accounts for the nonuniformity of velocities in each cross section. From equations 73,74 , and 75 ,

$$
\beta=\frac{1}{Q V_{x}} \int^{Q} v_{x} d Q \approx \frac{1}{Q V_{x}} \Sigma^{Q}\left(v_{x} \Delta Q\right) .
$$

The energy equation and the momentum equation describe the same state of motion, and they are derived from the same differential 
equation. They are independently useful, however, because the energy equation is independent of the external boundary shear, and the momentum equation is independent of the energy losses due to internal viscous shear. Thus, equations 65 and 75 can be shown to be equivalent only when the relationship between the boundary shear force and the internal, energy-dissipating viscous shear forces is explicit. This is true only when the flow is truly uniform (see below).

Many complex phenomena (such as the hydraulic jump, abrupt enlargements, and short transitions, all involving rapidly accelerated motion) are readily and accurately described by means of the momentum equation simply because the boundaries involved are short and the boundary shear forces can be ignored. The energy equation can be applied to these phenomena, too, but it includes a term of primary importance which compensates for the work done by the internal viscous shear forces. Ordinarily, the energy-loss term is evaluated by experimental means. However, because of their peculiar independence in these phenomena, the energy and the momentum equations can be solved simultaneously to yield analytical solutions for the energy loss.

\section{UNIFORM AND GRADUALLY VARIED FLOW}

\section{CHARACTERISTICS OF UNIFORM FLOW}

In most of the foregoing discussion, generality was maintained by assuming that the flow under consideration was accelerated. The specific problem of uniform flow, illustrated in plate $2(m, n)$, will be examined next.

By definition, the total convective acceleration at all points in a uniform flow is zero; in other words, $a_{s}=0$ and $a_{n}=0$. It follows that the velocity is constant along each streamline. Because velocity is a vector quantity, this implies that the streamlines are straight lines and, therefore, that the boundaries are prismatic.

Uniformity does not require that the velocities in a transverse section be equal. However, it does require that the transverse velocity distribution, which is a function of boundary drag and internal shear forces, be identical at all successive sections. As a corollary to the last requirement, uniformity implies that the rate of loss of energy is also constant. It follows that the average velocity and the velocity-head coefficient $(\alpha)$ are the same at successive sections. Thus, from the one-dimensional energy equation (65),

$$
h_{1}-h_{2}=-\Delta h=H_{t},
$$

which states that, as a distinctive characteristic of uniform flow, the loss of piezometric head (or the negative change of piezometric head) is equal to the loss of total energy head. 
Because the velocity pattern is uniform, the momentum coefficient $(\beta)$ and the momentum flux are also constant. Thus, from equation 75 ,

$$
\Sigma F_{s}=Q_{\rho} \beta_{2} V_{s_{2}}-Q_{\rho} \beta_{1} V_{s_{1}}=0 .
$$

Equation 78 states that, in a uniform fluid motion, the resultant of the external forces is zero.

Most of the uniform-flow characteristics described above are illustrated in plate $2(\mathrm{~m}, \mathrm{n})$ for both open and enclosed conduits. It is apparent that the most distinctive characteristic of uniform flow in open channels is the requirement that the axis of the channel be parallel to the energy and piezometric grade lines. This is because the piezometric line and the upper, free surface of the flow are coincident.

\section{THE CHEZT EQUATION}

The Chezy equation is the basis for many empirical equations for uniform flow in both open and enclosed conduits. One of its derivatives, the Manning equation, is widely accepted in the U. S. Geological Survey as a practical formula for open channels. As an equation of motion, the Chezy equation must be either a form of the energy equation (77) or the momentum equation (78). In fact, as will be shown, it can be considered to be both.

Most frequently, perhaps, the Chezy equation is derived from the one-dimensional momentum equation. Thus, from equation 78 , the resultant of the external forces acting on a free body of fluid in uniform motion is zero, or,

$$
\Sigma F_{s}=F_{p} \nrightarrow F_{g} \nrightarrow F_{\tau}=0 .
$$

In this equation $s$ denotes the direction of the total velocity vector. The effective forces in the $s$ direction are $F_{p}$, the force due to boundary pressure; $F_{g}$, the force due to weight; and $F_{\tau}$, the boundary shear force. From page 14 the sum of the pressure and weight forces on an element of volume $(\Delta s \Delta A)$ can be expressed as a function of the piezometric gradient. Thus,

$$
\mathrm{F}_{p} \nrightarrow \mathrm{F}_{g}=-\frac{d}{d s}(p+\gamma z) \Delta s \Delta A,
$$

or, over the finite volume of length $L$ and area $A$;

$$
F_{p}+F_{g}=-\Delta(p+\gamma z) A=-(\Delta h) \gamma A .
$$

From equations 79 and 80 , considering the fact that the boundary shear force is negative in the $s$ direction,

$$
F_{\tau}=-(\Delta h)_{\gamma} A .
$$


Dimensional analysis, substantiated by experiment, indicates that $F_{r}$ can be expressed by the relationship

$$
F_{\tau}=C_{\tau} \rho P L V^{2},
$$

in which $C_{r}$ is a dimensionless drag-force coefficient, a form of the Euler number and a function of the Reynolds number and boundary geometry, including roughness. $P$ is the perimeter of the wetted cross section.

From equations 81 and 82 ,

$$
-\Delta h=C_{\tau} \frac{\rho}{\gamma} \frac{P}{A} L V^{2}=C_{\tau} \frac{L V^{2}}{g R},
$$

in which $R=A / P$ is the hydraulic radius of the wetted cross section. If equation 83 is solved for velocity,

$$
V=\sqrt{\frac{g}{C_{\tau}}} \sqrt{R\left(\frac{-\Delta h}{L}\right)}
$$

The quantity $-\Delta h / L$ in this equation is the $L$ gradient of the piezometric head. This quantity is unfortunately described as the hydraulic "slope" (S). For uniform flow in open channels, of course, $S$ is also equivalent to the free-surface elevation gradient and the totalenergy head gradient. Thus, if $S$ is substituted for the piezometric head gradient and $C_{c h}$ is substituted for the coefficient radical in the right-hand member of equation 84 ,

$$
V=C_{c h} \sqrt{R S} \text {. }
$$

This is the Chezy equation.

From equations 84 and 85 ,

$$
C_{c h}=\sqrt{\frac{g}{C_{\tau}}}
$$

It is apparent that $C_{c h}$, unlike $C_{\tau}$, is not a dimensionless coefficient. It. is, nevertheless, a drag-force coefficient and a function of the Reynoldsnumber and boundary geometry.

\section{ALTHRNATE DFRIVATION OF THE CHFZY FQUATION}

An alternate derivation of the Chezy equation is based on the onedimensional energy equation. In equation 77 the head-loss term $\left(H_{\Sigma}\right)$ represents the amount of work done by the external forces in. overcoming the viscous resistance to fluid deformation. Thus, whether the flow be laminar or turbulent, uniform or nonuniform, theenergy equation must contain a term which represents the flow energy- 
dissipated by the internal viscous shear forces. It is customary to express this loss of total energy as a function of the mean kinetic energy of flow, or, in general,

$$
H_{L}=C_{L}\left(\frac{V^{2}}{2 g}\right)
$$

where $C_{\boldsymbol{L}}$ is a dimensionleșs energy-loss coefficient which, like $C_{\boldsymbol{\tau}}$, is a form of the Euler number and a function of the Reynolds number and boundary geometry. Unlike $C_{\tau}$, of course, $C_{L}$ is not a drag-force coefficient.

From equation 77 , as one of the most distinctive characteristics of uniform flow, $-\Delta h=H_{L}$. It follows that, for this condition, the independent momentum and energy relationships expressed by equations 83 and 87 are equivalent, or,

$$
C_{\tau}\left(\frac{L V^{2}}{g R}\right)=C_{L}\left(\frac{V^{2}}{2 g}\right)
$$

from which,

$$
C_{\tau}=\frac{C_{L}}{2}\left(\frac{R}{L}\right)
$$

From equations 86 and 89 , therefore,

$$
C_{c h}=\sqrt{\frac{g}{C_{\tau}}}=\sqrt{\frac{2 g}{C_{L}}\left(\frac{L}{R}\right)}
$$

from which it is concluded that the Chezy coefficient is proportional to either a drag-force coefficient or an energy-loss coefficient. Thus, the Chezy equation can be regarded as a form of either the momentum equation or the energy equation.

It is pertinent to observe that $\beta$ is not included in the boundarydrag equation (82) and $a$ is not included in the energy-loss equation (87). Thus, the influence of velocity distribution is apparently ignored in both of the alternate derivations of the Chezy equation. This procedure is justified by the observation that $\beta$ and $a$ are functions of the Reynolds number and boundary geometry-variables which also govern the value of $C_{r}$ in equation 82 and $C_{L}$ in equation 87. Thus, the Chezy coefficient takes full account of the influence of velocity distribution because it depends on the variables which govern the normal velocity pattern in uniform flow.

If a velocity nonuniformity is caused by an added variable, such as a local boundary irregularity, $a$ and $\beta$ will depend on this local condition as well as the normal resistance characteristics of the channel. For this condition, of course; the flow would not be uniform, the 
derivations leading to the Chezy equation would be invalid, and the question of including $a$ and $\beta$ in the Chezy equation is not pertinent.

\section{GRADUALLY VARIFD FLOW IN OPEN CHANNELS}

Truly uniform flow seldom occurs in open channels. Thus, most practical problems involving both artificial and natural channels are concerned with either rapidly varied or gradually varied nonuniform motion. In either kind of flow, the nonuniformity may represent either an acceleration or a deceleration. Accelerated fluid motion is characteristically stable, but decelerated motion, especially if it is rapidly varied, is usually accompanied by separation, extreme turbulence, and, therefore, a large energy loss.

The distinction between rapidly varied and gradually varied flow is conveniently associated with the relative magnitudes of the quantities $H_{L}$ and $F_{\tau}$ in the energy and momentum equations, respectively. A rapidly varied flow pattern, by definition, occurs in a relatively short length of channel. Thus, the boundary shear force $\left(F_{\tau}\right)$ is usually negligible, and the momentum equation, free of this troublesome term, provides an adequate solution for many rapidly varied flow problems. If the flow is accelerating as well as rapidly varied, the energy loss might also be negligible. For this condition the energy equation provides useful solutions. On the other hand, if the flow is rapidly varied and decelerating, the energy loss is usually of major importance, even when the boundary shear is negligible.

Gradually varied flow, by definition, occupies a comparatively long length of channel. Thus, both the boundary shear force and the energy loss are significantly related to the flow pattern. The two are explicitly related to each other only in the limiting condition of uniform flow, as described on page 49.

Between the extremes of rapidly varied flow and uniform flow, gradually varied flow in open channels is a complex function of many variables. Neither the boundary-shear mechanism nor the energyloss mechanism for this kind of flow is fully understood. In fact, neither $F_{\tau}$ nor $H_{L}$ can be satisfactorily evaluated.

In view of the foregoing arguments, it would appear to be ridiculous to propose that the Chezy equation, or any other uniform-flow equation, could be used to describe gradually varied flow in open channels. Nevertheless, lacking : a better solution, it is common practice to assume that a gradually varied nonuniform flow reach can be represented as the sum of constituent subreaches within which the flow is essentially uniform: : It is apparent that such reasoning leads to the conclusion that the energy-loss and boundary-shear mechanisms are the same in nonuniform flow as in uniform flow. This must surely be incorrect-and increasingly so as the nonuniformity increases. 
In general, the velocity distribution as well as the average velocity is different at successive cross sections in nonuniform flow. Thus, if the one-dimensional energy equation is applied to a gradually varied flow reach, the velocity-head coefficients at the end sections $\left(a_{1}\right.$ and $a_{2}$ ) are different. In addition, if an equation like the Chezy equation is used to evaluate the total head loss $\left(H_{L}\right)$ in a nonuniform reach it must be adjusted to account for the effect of channel shape and for the effect of using average values of the conveyance characteristics at the ends of the reach. In other words, if uniform-flow equations are to be used satisfactorily to describe a reach of gradually varied flow they must be adjusted to take into consideration the effect of various kinds and degrees of nonuniformity. These adjustments are ordinarily included in estimates of the Chezy coefficient, and the estimates are based on analyses of field and laboratory measurements made in a variety of open channels. Needless to say, the procedure is not dependable.

Application of the one-dimensional momentum equation (75) to a gradually varied flow is also complicated by changes in the velocity distribution at successive sections. Thus, the momentum coefficients $\left(\beta_{1}\right.$ and $\left.\beta_{2}\right)$ are different, and the value of the coefficient $C_{\tau}$ in equation 82 must be adjusted to account for the variations in the velocity pattern within the reach. Evaluation of boundary pressure forces is also complicated by the fact that the boundary is not prismatic. There is clearly no advantage in applying the nomentum equation instead of the energy equation to a gradually varied flow. 